\title{
VERZEICHNIS DER VERWENDETEN LITERATUR
}

\author{
Im Repertorium nicht aufgefiihte Quellen
}

Die Werke sind nach den mittelalterlichen Autoren geordnet. Es wird die Namensform aus Medioevo Latino verwendet, mit anderen geläufigen Namensformen in Klammern.

Abaelardus Petrus:

Orlandi, Giovanni (Hg.) / Marenbon, John (Einl.), Peter Abelard. Collationes, Oxford 2001.

Burnett, Charles, „Peter Abelard Soliloquium. A Critical Edition“, Studi medievali 25, 2 (1984), 857-894.

Accessus zu Ciceros De amicitia:

Pellegrin, Elisabeth, „Quelques accessus au De amicitia de Cicéron“, in: Cambier, Guy (Hg.), Hommages à André Boutemy, Bruxelles 1976, 274-298.

Adam Scotus (von Dryburgh), Soliloquium:

Bouvet, Jean, „Le Soliloquium d'Adam de Perseigne“, Collectanea Cisterciensia 50 (1988) 113-171 (mit falscher Zuschreibung).

Pez, Bernhard, Thesaurus anecdotorum novissimus, 1, 2, Augsburg 1721, 337-372 (ND in PL 198, 843-872).

Adelardus Bathoniensis (Adelard von Bath):

Burnett, Charles (Hg., Übers.), Adelard of Bath, Conversations with his Nephew. On the Same and the Different, Questions on Natural Science, and On Birds, unter Mitarbeit von Italo Ronca, Pedro Mantas España und Baudouin van den Abeele, Cambridge 1998.

Aelfricus Bata:

Gwara, Scott (Hg.) / Porter, David (Übers.), Anglo-Saxon Conversations. The Colloquies of Aelfric Bata, Woodbridge 1997.

Aelredus Rievallensis abbas (Aelred von Rievaulx), De spiritali amicitia:

Hoste, Anselm (Hg.), „De spiritali amicitia“ in: Hoste, Anselm / Talbot, Charles H. (Hgg.), Aelredi Rievallensis Opera omnia. I. Opera ascetica, CG CM 1, Turnhout 1971, 281-350.

Alanus ab Insulis (Alain de Lille), De fide catholica:

PL 210, 305-430.

Albertinus Mussatus:

Padrin, Luigi (Hg.) / Carducci, Giosue (Einl.), Albertino Mussato. Ecerinide: tragedia, Bologna 1900, ND Bologna 1969.

Berrigan, Joseph R. (Hg.), Mussato's Ecerinis and Loschi's Achilles, München 1975, ND der Editionen Padrin bzw. Schio mit englischer Übers. von Berrigan.

Müller, Hubert, Früher Humanismus in Oberitalien. Albertino Mussato: Ecerinis, Frankfurt a. Main et al. 1987, ND der Ed. Padrin mit Studie, deutscher Übersetzung und Kommentar von Müller.

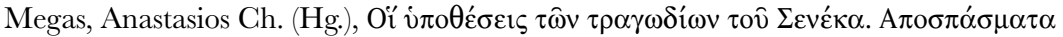

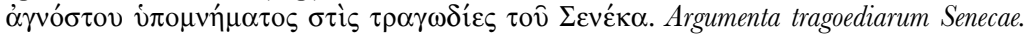
Commentarii in L. A. Senecae Tragoedias fragmenta nuper reperta, Thessalonica 1969.

\section{Alcuinus:}

Disputatio de vera philosophia, PL 101, 849-454.

De grammatica, PL 101, 854-902.

Dialogus de rhetorica et virtutibus, PL 101, 919-949.

De dialectica, PL 101, 949-976. 
Altercatio Aecclesie contra Synagogam:

Blumenkranz, Bernhard, „Altercatio Aecclesie contra Synagogam. Texte inédit du X $\mathrm{X}^{\mathrm{e}}$ siècle“, Revue du Moyen Age Latin 10 (1954), 5-159.

Altercatio duorum geometricorum de figuris, numeris et mensuris:

Folkerts, Menso, „Die Altercatio in der Geometrie I des Pseudo-Boethius. Ein Beitrag zur Geometrie im mittelalterlichen Quadrivium“, in: Keil, Gundolf (Hg.), Fachprosa-Studien. Beiträge zur mittelalterlichen Wissenschafts- und Geistesgeschichte, Berlin 1982, 84-114.

Altercatio Ecclesiae et Synagogae:

Hillgarth, Jocelyn N. (Hg.), Altercatio Ecclesiae et Synagogae / Conti, Marco (Hg.), Potamii episcopi Olisiponensis opera omnia, CG SL 69A, Turnhout 1999.

Altercatio Hadriani Augusti et Epicteti philosophi:

Daly, Lloyd William / Suchier, Walther (Hgg.), Altercatio Hadriani Augusti et Epicteti philosophi, Urbana (Illinois) 1939.

Siehe auch unter Ioca monachorum.

Anselmus Cantuariensis (Anselm von Canterbury):

Schmitt, Franciscus Salesius (Hg.), S. Anselmi Cantuariensis archiepiscopi Opera omnia, 6 Bde., Edinburgh 1946-1961.

Southern, Richard W. / Schmitt, Franz S. (Hgg.) Memorials of St. Anselm, Auctores Britannici Medii Aevi 1, Oxford 1969.

Anselmus Havelbergensis (Anselm von Havelberg):

Salet, Gaston (Hg.), Anselme de Havelberg. Dialogues. Livre I, Paris 1966.

Arnoldus Sancti Emmerami praepositus (Arnold von St. Emmeram):

Waitz, Georg (Hg.), „Ex Arnoldi libris de S. Emmeramo“, MGH script. in fol. 4, Hannover 1841, ND Stuttgart 1982, 543-574 (liber II, 555-574).

Augustinus:

Skutella, Martin (Hg.), Sancti Aureli Augustini Confessionum libri XIII, 2. von Wiebke Schaub und Heiko Jürgens korr. Auflage, Stuttgart 1981.

Hörmann, Wolfgang (Hg.), Sancti Aureli Augustini Opera. I, IV. Soliloquiorum libri duo. De inmortalitate animae. De quantitate animae, CSEL 89, Wien 1986.

Mutzenbecher, Almut (Hg.), Sancti Aureli Augustini Retractationum libri II, CG SL 57, Turnhout 1984.

Auxilius, Tractatus qui Infensor et Defensor dicitur:

PL 129, 1077-1102.

Beda Venerabilis:

Jones, Charles W. u. a. (Hgg.), Bedae Venerabilis Operae. VI. Opera didascalica, 3 Bde., CC SL 122-A, B und C, Turnhout 1975.

Benedictus Cassinensis abbas, Regula:

Hanslik, Rudolph (Hg.), Benedicti Regula, CSEL 75, Wien 1960.

Berengarius Turonensis (Berengar von Tours):

Beekenkamp, Willem H. (Hg.), Berengarii Turonensis De sacra coena adversus Lanfrancum, 's-Gravenhage 1941.

Bernardus Claraevallensis (Bernhard von Clairvaux):

Leclercq, Jean (Hg.), Bernardi Opera, 8 Bd., Roma 1957-1977.

Bernardus Claraevallensis?, Disputatio cuiusdam iusti cum Deo:

PL 184, 1155-1158.

Boethius:

Moreschini, Claudio (Hg.), Boethius. De consolatione Philosophiae. Opuscula theologica, 2. Edition, München / Leipzig 2005.

Bridlington-Dialog:

A Religious of C. S. M.V. [Sister Penelope Lawson] (Hg.), Robert of Bridlington, The Bridlington Dialogue: an Exposition of the Rule of St. Augustine for the Life of the Clergy, Given through a Dialogue between Master and Disciple, London 1960. 
Cassian (Johannes Cassianus), Collationes:

Petschenig, Michael, Iohannis Cassiani Conlationes XXIIII, CSEL 13, Wien 1886.

Clemens Scotus, Ars grammatica:

Tolkiehn, Joannes (Hg.), Clementis Ars grammatica, Philologi supplement. 20, 3, Leipzig 1928.

Conradus Hirsaugiensis mag. (Konrad von Hirsau):

Huygens, Robert B. C. (Hg.), Accessus ad auctores. Bernard d'Utrecht. Conrad d'Hirsau, Dialogus super auctores, Leiden 1970.

Bultot, Robert (Hg.), Dialogus de mundi contemptu uel amore attribué à Conrad d'Hirsau. Extraits de l'Allocutio ad Deum et du De veritatis inquisitione, Analecta Mediaevalia Namurcensia 19, Louvain / Lille 1966.

Conradus de Monte Puellarum (Konrad von Megenberg), Planctus ecclesiae in Germania: Scholz, Richard (Hg.), Die Werke des Konrad von Megenberg. 1. Planctus ecclesiae in Germania, MGH Staatsschriften des späteren Mittelalters 2, 1, Stuttgart 1941 (ND Stuttgart 1977).

De vita vere apostolica:

PL 170, 610-664.

Desiderius Casinensis abbas (Desiderius de Montecassino), Dialogi:

Schwartz, Gerhard / Hofmeister, Adolf (Hgg.), „,Dialogi de miraculis sancti Benedicti auctore Desiderio abbate Casinensi“", MGH script. in fol. 30, 2, Leipzig 1934, ND Stuttgart 1976, 1116-1151.

Dialog zwischen einem Säkular- und einem Regularkanoniker:

Fuchs, Franz / Märtl, Claudia, „Ein neuer Text zur Auseinandersetzung zwischen Säkular- und Regularkanonikern im 12. Jahrhundert", in: Mordek, Hubert (Hg.), Papsttum, Kirche und Recht im Mittelalter: Festschrift fir Horst Fuhrmann zum 65. Geburtstag, Tübingen 1991, 277-302.

Dialogus de pontificatu sanctae Romanae ecclesiae:

Böhmer, Heinrich (Hg.), Dialogus de pontificatu sanctae Romanae ecclesiae, MGH Libelli de lite 3, Hannover 1897, 526-546. Ergänzungen bei Deutinger, Rahewin, 219-222.

Dialogus de vita vere apostolica (Rupert von Deutz?):

PL 170, 609-664.

Dialogus inter Christianum et fudaeum (anonym, Alexander von Lincoln gewidmet):

PL 163, 1045-1072.

Disputatio cuiusdam iusti cum Deo:

PL 184, 1155-1158.

Donatus Ortigraphus:

Chittenden, John (Hg.), Donatus ortigraphus. Ars grammatica, Grammatici Hibernici Carolini aevi pars IV, CG CM 40D, Turnhout 1982.

Eberhardus Yprensis (Eberhard von Ypern), Dialogus:

Haring, Nicholas M., „A Latin Dialogue on the Doctrine of Gilbert of Poitiers“, Medieval Studies 15 (1953), 243-289.

Ermenricus Elwangensis (Ermenrich von Ellwangen), Vita Hariolf:

Pertz, Georg Heinrich (Hg.), „Vita Hariolfi fundatoris monasterii Ellwangensis auctore Ermenrico", MGH script. in fol. 10, Hannover 1852, ND 1987, 11-14.

Burr, Viktor (Hg.), „Vita Hariolf““, in: Burr, Viktor (Hg.), Ellwangen 764-1964. Beiträge und Untersuchungen zur 1200-Fahrfeier, Bd. 1, Ellwangen 1964, 14-31.

Eugenius Vulgarius, Liber cuiusdam requirentis et respondentis:

PL 129, 1103-1112 (hier Auxilius zugeschrieben).

Galandus Regniacensis (Galand von Reigny), Parabolarium:

Friedlander, Colette / Leclercq, Jean / Raciti, Gaetano (Hgg.), Galand de Reigny. Parabolaire, Sources Chrétiennes 378, Paris 1992.

Gerhohus Reicherspergensis (Gerhoch von Reichersberg), Epistola ad Innocentium papam:

Sackur, Ernst (Hg.), Epistola ad Innocentium papam missa quid distet inter clericos seculares et 
regulares, MGH Libelli de lite 3, Hannover 1897, ND Hannover 1956, 202-239

(Ergänzungen aus der Wiener Hs. bei Van den Eynde, L'œuvre, 333-339).

Gervasius Melkeleius (Gervasius von Melkley), Ars poetica:

Gräbener, Hans-Jürgen (Hg.), Gervais von Melkley. Ars poetica, Münster 1065.

Gilbertus Crispinus:

Abulafia, Anna Sapir / Evans, G. R., The Works of Gilbert Crispin Abbot of Westminster, Auctores Britannici Medii Aevi 8, London 1986.

Glossaria:

Goetz, Georg (Hg.), Corpus Glossariorum Latinorum, Bd. 1-7, Leipzig 1888-1923, ND Amsterdam 1965.

Glossaria Latina iussu Academiae Britannicae editae. Bd. 1. Glossarium Ansileubi sive librum glossarum, ed. Wallace M. Lindsay, J.-F. Mountford, J. Whatmough, etiam F. Rees, R. Weir, M. Laistner, Paris 1926, ND Hildesheim 1965.

Glossaria Latina iussu Academiae Britannicae editae. Bd. 2. Arma, Abavus, Philoxenus, edd. Wallace M. Lindsay, R.-G. Austin, M. Laistner, J. F. Mountford, Paris 1926, ND Hildesheim 1965

Glossaria Latina iussu Academiae Britannicae editae. Bd 3. Abstrusa, Abolita, ed. Wallace M. Lindsay, H.-J. Thomson, Paris 1926, ND Hildesheim 1965.

Glossaria Latina iussu Academiae Britannicae editae. Bd. 4. Placidus, Festus, edd. J. W. Pirie / Wallace M. Lindsay, Paris 1930, ND Hildesheim 1965.

Glossaria Latina iussu Academiae Britannicae editae. Bd. 5. Abba, AA, edd. Theander, Carl / M. Inguanez, C.-J. Fordyce, Paris 1931, ND Hildesheim 1965.

Gregorius Magnus:

Moricca, Umberto (Hg.), Gregorii Magni Dialogi libri IV, Roma 1924.

Mauristen (Dom Denys de Sainte-Marthe), Sancti Gregorii Papae I... Opera omnia, Paris 1705, abgedruckt in Migne PL 77, 149-430.

De Vogüé, Adalbert (Hg.), Grégoire le Grand. Dialogues, 3 Bde., Paris 1978-1980.

Guido Ferrariensis ep. (Wido von Ferrara), De scismate Hildebrandi:

Dümmler, Ernst (Hg.), De scismate Hildebrandi. Pro illo et contra illum, Libelli de lite 1, Hannover 1891, ND Hannover 1956, 529-567 (Ergänzungen in MGH Libelli de lite 3, Hannover 1897, 731-733).

Guillelmus Alfachinus Ianuensis (Guglielmo Alfachino):

Limor, Ora, Die Disputationen zu Ceuta (1179) und Mallorca (1286). Zwei antijüdische Schriften aus dem mittelalterlichen Genua, MGH Quellen zur Geistesgeschichte des Mittelalters 15, München 1994.

Guillelmus de Aragonia (Wilhelm von Aragon):

Crespo, Roberto, „Il prologo alla traduzione della ,Consolatio Philosophiae“ di Jean de Meun e il commento di Guglielmo d'Aragona“, in: den Boer, Willem / van der Nat, Pieter G. / Sicking, Christiaan M. J. / van Winden, J. C. M. (Hgg.), Romanitas et Christianitas. Studia Iano Henrico Waszink A. VI Kal. Nov. A. MCMLXXIII XIII lustra complenti oblata, Amsterdam / London 1973, 55-70.

Guillelmus de Conchis (Wilhelm von Conches):

Jeauneau, Édouard (Hg.), Guillaume de Conches. Glosae super Platonem, Paris 1965.

Nauta, Lodi (Hg.), Guillelmi de Conchis Glosae super Boetium, CG CM 158, Turnhout 1999.

Ronca, Italo (Hg.), Guillelmi de Conchis Dragmaticon Philosophiae, GC CM 152, Turnhout 1997.

Guillelmus de Sarzano (Wilhelm von Sarzano), Tractatus de potestate summi pontificis:

Del Ponte, Renato (Hg.) / Capitani, Ovidio (Einf.), „Il Tractatus de potestate Summi Pontificis die Guglielmo da Sarzano“, Studi medievali 3, 12 (1971), 997-1094.

Miethke, Jürgen (Hg.), „Ein neuer Text zur Geschichte der politischen Theorie im 14. Jahrhundert: Der Tractatus de potestate summi pontificis des Guillelmus de Sarzano aus Genua“, Quellen und Forschungen aus italienischen Archiven und Bibliotheken 4 (1974), 509-538. 
Guillelmus Hirsaugiensis abbas (Wilhelm von Hirsau):

Astronomica, in: Pez, Bernhard (Hg.), Thesaurus anecdotorum novissimus, Bd. 6, 1, Augsburg 1729, 259-264, danach PL 150, 1639-1642 (an beiden Orten nur die Praefatio).

Harbinson, Denis (Hg.), Wilhelmi Hirsaugiensis Musica, Corpus scriptorum de musica 23, Roma 1975.

Guillelmus Sancti Dionisii monachus (Wilhelm von Saint-Denis):

Wilmart, André, „Le dialogue apologétique du moine Guillaume, biographe de Suger", Revue Mabillon 32 (1942), 80-118.

Henricus Septimellensis (Heinrich von Settimello), Elegia:

Cremaschi, Giovanni (Hg.), Enrico da Settimello. Elegia, Bergamo 1949.

Herbordus Montis Sancti Michaelis Bambergensis scholasticus (Herbord von Michelsberg), Dialogus de vita Ottonis:

Köpke, Rudolf (Hg.), „Dialogus de vita Ottonis episcopus Babenbergensis“, MGH script. in fol. 20, Hannover 1868, ND Stuttgart 1989, 697-771.

Köpke, Rudolf (Hg.), Herbordi Dialogus de vita Ottonis episcopus Babenbergensis, MGH script. in us. schol. [33], Hannover 1868, 13-228.

Wikarjak, Ioannes (Hg.) / Liman, Casimirus (Einf., Komm.), Herbordi Dialogus de vita s. Ottonis episcopi Babenbergensis, Monumenta Poloniae historica 7, 3, Warszawa 1974.

Hildebertus Cenomanensis ep. (Hildebert von Lavardin), De querimonia:

Orth, Peter (Hg.), Hildeberts Prosimetrum De Querimonia und die Gedichte eines Anonymus. Untersuchungen und kritische Editionen, Wien 2000.

Honorius Augustodunensis:

Garrigues, Marie-Odile, „Honorius Augustodunensis. De esu volatilium“, Studia monastica 28 (1986), 75-130.

Lefèvre, Yves, L'Elucidarium et les Lucidaires: contribution, par l'histoire d'un texte, à l'histoire des croyances religieuses en France au Moven Âge, Paris 1954.

Lucentini, Paolo (Hg.), Honorius Augustodunensis. Clavis physicae, Roma 1974.

Hrabanus Maurus, De computo:

Stevens, Wesley M., Rabani Mogontiacensis episcopi De computo, in: Rabani Mauri Martyrologium. De computo, CG GM 44, Turnhout 1979, 165-331.

Hugo de Sancto Victore:

Baron, Roger (Hg.), Hugonis de Sancto Victore Opera propaedeutica. Practica geometriae, De grammatica, Epitome Dindimi in philosophiam, Notre Dame (Indiana) 1966.

Buttimer, Charles Henry (Hg.), Hugonis de Sancto Victore Didascalicon de studio legendi. A critical text, Washington 1939.

Feiss, Hugh B. / Sicard, Patrice / Poirel, Dominique / Rochais, Henri, L'œuvre d'Hugues de Saint-Victor. 1. De institutione novitiorum. De virtute orandi. De laude caritatis. De arrha animae, Turnhout 1997.

Müller, Karl, Hugo von St. Victor. Soliloquium de arrha animae und De vanitate mundi, Bonn 1913.

Sicard, Patrice (Hg.), Hugonis de Sancto Victore De archa Noe. Libellus de formatione arche, CC CM 176, Turnhout 2001.

PL 176.

Iacobus de Theramo, Belial:

M. G. H. (Melchior Goldast Hamesfeldiensis?), Processus iuris ioco-serius, Hanoviae 1611.

Idungus monachus:

Huygens, Robert B. C., Le moine Idung et ses deux ouvrages, „Argumentum super quatuor questionibus" et „Dialogus duorum monachorum“, Spoleto 1980 (erweiterte Einführung und Text der Erstveröffentlichung: „Le moine Idung et ses deux ouvrages, Argumentum super quatuor questionibus et Dialogus duorum monachorum", Studi Medievali 13, 1 (1972), 291-470). 
Ioachim de Flore, Dialogi de prescientia Dei et predestinatione electorum:

Potestà, Gian Luca (Hg.), Ioachim abbas Florensis. Dialogi de prescientia Dei et predestinatione electorum, Ioachim abbas Florensis. Opera omnia. IV, 1, Roma 1995.

Ioca monachorum:

Suchier, Walther (Hg.), Das mittellateinische Gespräch Adrian und Epictitus nebst verwandten Texten (Foca Monachorum), Tübingen 1955.

Iohannes Burgundi (Joan Burgunyó):

Madurell Marimón, José María, „,Juan Burgunyó, embajador de Jaime II“, Analecta Sacra Tarraconensia 15 (1942), 265-289, Text 282-289.

Iohannes Ianuensis de Balbis (Johannes Balbus), Catholicon:

Catholicon, unveränd. ND der Ausgabe Mainz 1460 (Hain *2254), Peterborough 1971.

Iohannes Scottus Eriugena, Periphyseon:

Jeauneau, Édouard A. (Hg.), Iohannis Scotti seu Eriugenae Periphyseon, 5 Bde., CG CM 161-165, Turnhout 1996-2003.

Isidorus Hispalensis ep. (Isidor von Sevilla), Origines:

Lindsay, Wallace M. (Hg.), Isidori Hispalensis episcopi Etymologiarum sive Originum libri $X X$, Oxford 1911.

Lanfrancus Cantuariensis archiep., Liber de corpore et sanguine Domini:

Martello, Concetto (Hg.), Lanfranco contro Berengario nel Liber de corpore et sanguine Domini, Catania 2001.

Huygens, Robert B. G. (Hg.), „Bérenger, Lanfranc, Bernold“, Sacris erudiri 16 (1965), 355-403 (Exzerpte, 370-377, zur Überlieferung 358-368). ND in Huygens, Robert B. C. (Hg.), Serta Mediaevalia. Textus varii saeculorum X-XIII in unum collecti, CC CM 171, Turnhout 2000 (Exzerpte 239-246, zur Überlieferung 227-238).

Liber Quare:

Götz, Georg Polycarp (Hg.), Liber Quare, GC CM 60, Turnhout 1983.

Liutprandus Cremonensis ep.:

Chiesa, Paolo (Hg.), Liutprandi Cremonensis opera omnia, CG CM 156, Turnhout 1998

Malcalanus, Dialogus de statu sanctae ecclesiae:

Löwe, Heinz, ,Dialogus de statu sanctae ecclesiae. Das Werk eines Iren im Laon des 10. Jahrhunderts", Deutsches Archiv 17 (1961), 12-90.

Martinus de Leibitz, Trialogi:

Jellouschek, Carolus Johannes (Hg.), Trialogi ascetici, quibus accedunt Sermo in monasteriorum visitatione factus et Caeremonialia et Quotlibetarum, Padova 1932.

Nicolaus Cusanus, Trialogus de possest:

Steiger, Renata (Hg.), Nicolai de Cusa. Trialogus de possest, Nicolai de Cusa Opera Omnia 11, 2, Hamburg 1973.

Nicolaus Dresdensis, De purgatorio:

De Vooght, Paul (Hg.), „Le dialogue De purgatorio (1415) de Nicolas de Dresde“, Recherches de Théologie ancienne et médiévale 42 (1975), 132-223.

Odo Cameracensis ep. (Odo von Cambrai):

PL 160, 1103-1112, ND mit deutscher Übersetzung in: Bunte, Wolfgang, Religionsgespräche zwischen Christen und Fuden in den Niederlanden (1100-1500), Frankfurt am Main et al. 1990, 17-49.

Papias:

Papias, Vocabulista, Venedig 1496, ND Torino 1966.

Paschalis Romanus, Disputatio contra fudeos:

Dahan, Gilbert, „Paschalis Romanus. Disputatio contra Fudeos“, Recherches augustiniennes 10 (1975), 161-213.

Paulus Burgensis, Scrutinium scripturarum:

Scrutinium scripturarum, Druck Mainz: Peter Schöffer, 1478 (HC 10766), konsultiert in dem Exemplar München, BSB, 2 Inc.c.a. 765. 
Petrus Alphonsi:

Mieth, Klaus Peter, Der Dialog des Petrus Alfonsi, seine Überlieferung im Druck und in den Handschriften; Textedition, Diss. Freie Universität Berlin, Berlin 1982.

Mieth, Klaus Peter (Hg.) / Ducay, Esperanza (Übers.) / Tolan, John (Einf.), Pedro Alfonso de Huesca. Diálogo contra fudios, Huesca 1996.

Hilka, Alfons / Söderhjelm, Werner (Hgg.), Die Disciplina clericalis des Petrus Alfonsi (das älteste Novellenbuch des Mittelalters), Sammlung mittellateinischer Texte 1, Heidelberg 1911.

Hilka, Alfons / Söderhjelm, Werner (Hgg.), Petri Alfonsi Disciplina clericalis, Helsingfors 1911.

Petrus Blesensis (Petrus von Blois), Dialogus inter regem Henricum et abbatem Bonevallis:

Huygens, Robert B. C., „Pierre de Blois. Dialogus inter regem Henricum et abbatem Bonevallis", Revue bénédictine 68 (1958), 87-112. Jetzt in: Huygens, Robert B. C., Serta mediaevalia. Textus varii saeculorum $x-x$ xii in unum collecti. Tractatus et epistulae, CG CM 171, Turnhout 2000, 374-408.

Petrus Damiani:

Antilogus contra judaeos, PL 145, col. 41-57.

Heinemann, Lothar von (Hg.), Disceptatio sinodalis, MGH Libelli de lite 1, Hannover 1891, ND Hannover 1956, 77-94.

Reindel, Kurt, Die Briefe des Petrus Damiani. Teil 2, MGH Epp. Briefe der deutschen Kaiserzeit 4, 2, München 1988, Nr. 89, 531-572.

Petrus Helias, Summa super Priscianum:

Reilly, Leo (Hg.), Petrus Helias. Summa super Priscianum, Bd. 1 und 2, Toronto 1993.

Pius II papa (Enea Silvio Piccolomini), Pentalogus de rebus ecclesiae et imperii:

Pez, Bernhard (Hg.), Thesaurus Anecdotorum novissimus, Bd. 4, 3, 637-744.

Kritische Edition von Christoph Schingnitz in Vorbereitung für MGH.

Plato Latinus, Timaeus:

Waszink, Jan Hendrik (Hg.), Plato Latinus. Vol. IV. Timaeus a Calcidio translatus commentarioque instructus, London 1962.

Ps. Augustinus, Soliloquia animae ad Deum:

PL 40, 863-898.

Ps. Bernhard, Soliloquium:

PL 184, 1158-1168.

Quaestiones de iuris subtilitatibus:

Zanetti, Ginevra (Hg.), Questiones de iuris subtilitatibus, Firenze 1958.

Ratherius Veronensis, Dialogus confessionalis:

Reed, Peter L. D., Ratherii Veronensis Opera, CG CM 46A, Turnhout 1984, 219265.

Radulphus Sancti Trudonis abbas (Rudolf von St. Trond), De virginitate beatae Mariae:

Boutemy, André, „Carmina Trudonensia“, Mélanges Foseph de Ghellinck S.F. Tome II. Moyen Âge, Époques moderne et contemporaine, Gembloux 1951, 583-601.

Richardus filius Nigelli (FitzNigel, von Ely), Dialogus de scaccario:

Johnson, Charles (Hg., Übers.), Dialogus de Scaccario. The Course of the Exchequer by Richard FitzNigel and Constitutio Domus Regiae. The Establishment of the Royal Household, with corrections by F. E. L. Garter and Diana E. Greenway, Oxford 1983.

Richardus de Sancto Victore, De Emmanuele:

De Emmanuele libri II, PL 196, 601-666.

Rogerius:

Kantorowicz, Hermann, Studies in the Glossators of the Roman Law. Newly discovered writings of the twelfth century, unter Mitarbeit von William Warwick Buckland, Cambridge 1938, ND mit Addenda et Corrigenda von Peter Weimar, Aalen 1969.

Rupertus Tuitiensis abbas (Rupert von Deutz):

Altercatio monachi et clerici quod liceat monacho praedicare, PL 170, 537-542. 
Arduini, Maria Lodovica / Haacke, Rhabanus, Ruperto di Deutz e la controversia tra Cristiani ed Ebrei nel secolo XII. Con testo critico dell'Anulus seu dialogus inter Christianum et Judaeum a cura di Rhabanus Haacke, Roma 1979. Lateinischer Text abgedruckt mit deutscher Übersetzung in: Bunte, Wolfgang, Religionsgespräche zwischen Christen und Juden in den Niederlanden (1100-1500), Frankfurt am Main et al. 1990, 103-262.

Salomon et Marcolfus:

Benary, Walter (Hg.), Salomon et Marcolfus, Heidelberg 1914.

Seneca:

Vottero, Dionigi (Hg.), Lucio Anneo Seneca. I Frammenti, Bologna 1998.

Newman, Robert Joseph, Lucii Annaei Senecae De remediis fortuitorum liber ad Gallionem fratrem, Diss. The Johns Hopkins University (Baltimore, Maryland) 1984.

Speculum virginum:

Seyfarth, Jutta (Hg.), Speculum virginum, CG CM 5, Turnhout 1990.

Seyfarth, Jutta (Hg., Übers.), Fungfrauenspiegel, 4 Bde., Fontes cristiani 30, Freiburg et al. 2001.

Stephan von Olmütz, Dialogus volatilis inter aucam et passerem adversus Hussum:

Pez, Bernhard, Thesaurus anecdotorum novissimus, Bd. 4, Augsburg 1723, 431-502.

Sulpicius Severus:

Halm, Carolus (Hg.), Sulpicii Severi Libri qui supersunt, CSEL 1, Wien 1866.

Thomas de Chobham:

Morenzoni, Franco (Hg.), Thomas de Chobham. Sermones, CG CM 82ª, Turnhout 1993.

Varro (M. Terentius Varro Reatinus), Saturarum Menippearum fragmenta:

Cèbe, Jean-Pierre, Varron. Satires Ménippées. Édition, traduction et commentaire, Bd. 1-13, Roma 1972-1999.

Vita Beatae Virginis Mariae et Salvatoris rhythmica:

Vögtlin, Adolf (Hg.), Vita beatae virginis mariae et salvatoris rhythmica, Tübingen 1988.

Vocabularius Ex quo:

Grubmüller, Klaus / Schnell, Bernhard / Stahl, Hans-Jürgen / Auer, Erltraud / Pawis, Reinhard (Hgg.), ,Vocabularius Ex quo`. Überlieferungsgeschichtliche Ausgabe, 6 Bde., Tübingen 1988-2001.

Warnerius Basiliensis clericus:

Hoogterp, Pierre (Hg.), „Warnerii Basiliensis Paraclitus et Synodus“, Archives d'Histoire doctrinale et littéraire du Moyen Age 8 (1933), 261-433.

Wernher von Regensburg, Liber soliloquiorum:

Pez, Bernhard, Bibliotheca ascetica antiquo-nova. Bd. 4, Regensburg 1724 (ND Farnborough, Hants. 1967), 41-84.

Wipo, Tetralogus:

Bresslau, Harry (Hg.), Die Werke Wipos, MGH script. in us. schol. [61], Hannover / Leipzig 1915 (ND 1977), 75-86.

\section{Sekundärliteratur}

Hier wird die Sekundärliteratur aufgelistet, die in der Studie zitiert wurde, nicht jedoch diejenige, die nur im Repertorium verwendet wurde. Diese findet sich separat in jedem Repertoriumseintrag unter „Literatur“.

Da die Sekundärliteratur in abgekürzter Form mit den ersten Worten des Titels zitiert wird, sind die Werke eines Autors nicht nach dem Erscheinungsjahr, sondern alphabetisch nach dem Titel geordnet.

Abulafia, Anna Sapir, „Christians and Jews in the High Middle Ages: Christian Views of Jews", in: Cluse, Christoph (Hg.), The Jewes of Europe in the Middle Ages (tenth to fifteenth centuries), Turnhout 2004, 19-28. 
, Christians and Jewes in the twelfth-century renaissance, London / New York 1995.

, „Twelfth-Century Humanism and the Jews“, in: Limor, Ora / Stroumsa, Guy (Hgg.), Contra Iudaeos: Ancient and Medieval Polemics between Christians and Jewes, Tübingen 1996, 161-175, jetzt auch in: Abulafia, Christians and Jeres in Dispute, Aldershot 1998, art. XIV.

Adamzik, Kirsten, Textsorten-Texttypologie. Eine kommentierte Bibliographie, Münster 1995.

Albert, Mary, „The Better Paths of Wisdom. Alcuin's Monastic True Philosophy and the Worldly Court", Speculum 76, 2 (2001), 896-910.

Anton, Hans Hubert, Fürstenspiegel und Herrscherethos in der Karolingerzeit, Bonn 1968.

— , „Petrarca und die Tradition der Herrscher- und Fürstenspiegel“, in: Giebmeyer, Angela / Schnabel-Schüle, Helga (Hgg.), ,Das Wichtigste ist der Mensch. “ Festschrift für Klaus Gerteis zum 60. Geburtstag, Mainz 2000, 229-251.

Arduini, Maria Lodovica, ,, ,Sola ratione'. Le fonti, II. Il ricorrere del segno semantico ,sola ratione‘ in alcuni Autori della Cristianità latina: da Prudenzio ad Aelredo di Rievaulx. Per la conclusione dei ,Prolegomena' alla Edizione del ,Dialogus quae sit vita vere apostolica“", Sacris erudiri 37 (1997), 203-331.

_, „Un testo ancora da scoprire: il de vita vere apostolica. Prolegomena a l'edizione critica per il Corpus Christianorum e ipotesi interpretativa per l'attribuzione dell'opera“, in: L'Europa dei secoli XI e XII fra novità e tradizione: sviluppi di una cultura (Miscellanea del Centro di Studi Medioevali 12, Milano 1990), 305-377.

Auer, Albert, Fohannes von Dambach und die Trostbücher vom 11. bis zum 16. Fahrhundert, Münster 1928.

Aygon, Jean-Pierre, „Le dialogue comme genre dans la rhétorique antique“, Pallas 59 (2002) = Mélanges Fean Soubiran, 197-208.

Bachtin, Michail M., „Aus der Vorgeschichte des Romanwortes“, in: Bachtin, Die Ästhetik, 301-337 (deutsche Übersetzung, russisch 1940 erschienen).

__ , „Das Wort im Roman“, in: Bachtin, Die Ästhetik, 154-300 (deutsche Übersetzung, russisch 1934/35 erschienen).

—_, Die Ästhetik des Wortes, hg. und eingeleitet von Rainer Grübel, Frankfurt a. M. 1979

—_, Probleme der Poetik Dostoevskijs, Frankfurt a. M. / Berlin / Wien 1985.

- , ,The Problem of the Text in Linguistics, Philology, and the Human Sciences: An Experiment in Philosophical Analysis", in: Bachtin, Michail, Speech Genres and Other Late Essays, Austin 1986 (russisch 1976).

Badia, Lola, „A propòsit de Ramon Llull i la gramàtica“, in: Badia, Teoria i practica, 173-194 (zuerst erschienen in: Estudis de Llengua i Literatura Catalanes 18 (1989), 157-182).

— , „Estudi del Phantasticus de Ramon Llull“, in: Badia, Teoria i pràctica 31-53 (zuerst erschienen in: Estudios Lulianos 26 (1986), 5-22).

_ , „La novel-la espiritual de Barlaam i Josafat en el rerafons de la literatura lul-liana“, in: Badia, Teoria i pràctica, 97-119 (jetzt auch in: Catalan Review 4 (1990), 127-154).

—_, „Ramon Llull i la tradició literaria“, in: Badia, Teoria i practica, 73-95 (zuerst erschienen in: Estudios Lulianos 28 (1988), 121-138).

- Teoria i pràctica de la literatura en Ramon Llull, Barcelona 1991.

Badilita, Christian, „Les Apophthegmata Patrum“, in: Comunicazione e ricezione, 573-584.

Baldus, Gisela, Die Gestalt des „,dieners“ im Werke Heinrich Seuses, Diss. Köln 1966.

Baldwin, John W., „Philippe Auguste, Pierre le Chantre et Étienne de Gallardon: la conjoncture de regnum, studium et cancellaria au tournant des $\mathrm{XII}^{\mathrm{e}}$ et $\mathrm{XIII}^{\mathrm{e}}$ siècles", Académie des Inscriptions \& Belles Lettres. Comptes rendus des séances, 2000, fasc. 1, JanvierMars, 437-457.

Baldzuhn, Michael, „Schulunterricht und Verschriftlichungsprozess. Forschungsansätze und Forschungsergebnisse“, in: Meier, Christel / Honemann, Volker / Keller, Hagen / Suntrup, Rudolf (Hgg.), Pragmatische Dimensionen mittelalterlicher Schriftkultur (Akten des Internationalen Kolloquiums 26.-29. Mai 1999), München 2002, 161-175. 
Bardy, Gustave, Art. „Dialog. B. Christlich“, Reallexikon fir Antike und Christentum, Bd. 3 (1957), 945-955.

—_, „La littérature patristique des quaestiones et responsiones sur l'Écriture sainte“, Revue biblique 41 (1932), 210-236, 341-369, 515-537 und 42 (1933), 14-30, 211-229, 328-352.

Baron, Hans, Petrarch's Secretum. Its Making and its Meaning, Cambridge (Mass.) 1985.

Bauer, Renate, Adversus Fudaeos. Fuden und Fudentum im Spiegel alt- und mittelenglischer Texte, Frankfurt a. M. u. a. 2003.

Bayless, Martha, „Alcuin's Disputatio Pippini and the early medieval riddle tradition“, in: Halsall, Humour, 157-178.

Bazàn, Bernardo C. / Wippel, John W. / Fransen, Gérard / Jacquart, Danielle, Les questions disputées et les questions quodlibétiques dans les facultés de Théologie, de Droit et de Médecine, Turnhout 1985.

Beatrice, Pier Franco, Art. „Dialogo“, Dizionario patristico e di antichità cristiane, Bd. 1 (1983), 939-942.

Berger, David, „Gilbert Crispin, Alan of Lille and Jacob ben Reuben. A Study in the Transmission of medieval Polemic“, Speculum 49 (1974), 34-47.

Berges, Wilhelm, Die Fürstenspiegel des hohen und späten Mittelalters, Leipzig 1938.

Berlioz, Jacques / Polo de Beaulieu, Marie-Anne, Les Exempla médiévaux. Introduction à la recherche, suivie des tables critiques de l'Index exemplorum de Frederic C. Tubach, Carcassonne 1992.

— , „Les recueils d'exempla et la diffusion de l'encyclopédisme médiéval“, in: Picone, Michelangelo (Hg.), L'enciclopedismo medievale, Ravenna 1994, 179-212.

Berlioz, Jacques, Rezension von Ruelle, Le Dialogue in Romania 106 (1985), 273-285.

Bernabei, Richard, The Treatment of Sources in Macrobius' Saturnalia, and the Influence of the Saturnalia during the Middle Ages, Diss. Cornell University 1970.

Bernardo, Aldo S., „Dramatic Dialogue and Monologue in Petrarch's Works - II“, Symposion 7, 1 (1953), 92-119.

—, „Dramatic Dialogue in the Prose Letters of Petrarch“, Symposion 5, 2 (1951), 302-316.

Bernt, Günter, Art. „Dialog. IV. Lateinisches Mittelalter“, LMA 3 (1986), 951-955.

—, „Trostbücher. I. Mittellateinische Literatur“, LMA 8 (1997), 1048-1049.

Berschin, Walter, Biographie und Epochenstil im lateinischen Mittelalter. 5 Bde., Stuttgart 1986-2004.

Bertaud, Émile, Art. „Dialogues spirituels“, DictSpir 3 (1957), 834-850.

, ,Entretiens spirituels“, DictSpir 4 (1961), 763-774.

Betten, Anne, „Analyse literarischer Dialoge“, in: Fritz / Hundsnurscher 1994, 519-544.

Billanovich, Guido, „Abbozzi e postille del Mussato nel Vaticano lat. 1769“, Italia Medioevale e Umanistica 28 (1985), 7-35.

Billanovich, Guido / Travaglia, Guglielmo, ,Per l'edizione del De lite inter Naturam et Fortunam e del Contra casus fortuitos di Albertino Mussato", Bollettino del Museo Civico di Padova 31-43 (1942-1954), 279-296.

Binkley, Peter, „Debates and Dialogues“, in: Mantello / Rigg, 677-681.

Blumenkranz, Bernard / Châtillon, Jean, „De la polémique antijuive à la catéchèse chrétienne. L'objet, le contenu et les sources d'une anonyme Altercatio Synagogae et Ecclesiae du XII ${ }^{\mathrm{e}}$ siècle“, Recherches de théologie ancienne et médiévale 23 (1956), 40-60.

Bobes Naves, María del Carmen, El diálogo. Estudio pragmático, lingüístico y literario, Madrid 1992.

Bodemann, Ulrike / Grubmüller, Klaus, „Schriftliche Anleitung zu mündlicher Kommunikation: die Schülergesprächsbüchlein des späten Mittelalters", in: Keller, Hagen / Grubmüller, Klaus / Staubach, Nikolaus (Hgg.), Pragmatische Schriftlichkeit im Mittelalter. Erscheinungsformen und Entwicklungsstufen, München 1992, 177-193. 
Boesch Gajano, Sofia, „La proposta agiografica dei Dialogi di Gregorio Magno, Studi medievali III, 21 (1980), 623-664.

_ _, „Narratio e expositio nei Dialoghi di Gregorio Magno“, Bullettino dell'Istituto Storico Italiano per il Medio Evo e Archivio Muratoriano 88 (1979), 1-33.

Bolzoni, Lina, „Petrarca e le tecniche della memoria (a proposito del De remediis)“, in: Meroi, Fabrizio / Scapparone, Elisabetta (Hgg.), Humanistica. Per Cesare Vasoli, Firenze 2004, 41-60.

Bonman, Ottokar, „Wernher von Regensburg und sein Liber soliloquiorum“, Zeitschrift fiur Askese und Mystik 12 (1937), 294-405.

Bonner, Anthony / Badia, Lola, Ramon Llull. Vida, pensament $i$ obra literaria, Barcelona 1988.

Borst, Arno, Die Katharer, Schriften der Monumenta Germaniae Historica 12, Stuttgart, 1953.

Boschung, Peter, „Boethius and the early medieval Quaestio“, Recherches de Théologie et Philosophie médiévales 71 (2004), 233-259.

Bremond, Claude / Le Goff, Jacques / Schmitt, Jean-Claude, L'rexemplum, Typologie des Sources du Moyen Âge Occidental 40, Turnhout 1982.

Briesemeister, Dietrich, „Humanistische Dialoge in Spanien im Übergang zur Frühen Neuzeit", in: Guthmüller / Müller, 183-202.

Brinker, Klaus / Antos, Gerd / Heinemann, Wolfgang / Sager, Sven F., Text- und Gesprächslinguistik. Linguistis of Text and Conversation, Bd. 1, Berlin 2000.

Brückner, Annemarie, Art. „Dialogus creaturarum“, Enzyklopädie des Märchens 3 (1979), 601-604.

Brunhölzl, Franz, „Der Bildungsauftrag der Hofschule“, in: Bischoff, Bernhard (Hg.), Karl der Große. Band 2: Das geistige Leben, Düsseldorf 1965, 28-41.

—_, „Die lateinische Literatur“, in: Erzgräber, Willi (Hg.), Neues Handbuch der Literaturwissenschaft. Bd. 6. Europäisches Spätmittelalter, Wiesbaden 1978, 519-563.

__ Geschichte der lateinischen Literatur des Mittelalters, Bd. 1 u. 2, München 19751992.

Buck, August (Hg.), Petrarca, Darmstadt 1976.

Bürkle, Horst, Art. „Dialog der Religionen“, Lexikon für Theologie und Kirche, Bd. 3 (1995), 196-197.

Bussières, Marie-Pierre, „Conclusions: Questions (encore) sans réponses“, in: Volgers / Zamagni, 181-189.

Camerer, Luitgard, Die Bibliothek des Franziskanerklosters in Braunschweig, Braunschweig 1982.

Cameron, Averil, „Disputations, Polemical Literature and the Formation of Opinion in the Early Byzantine Period“, in: Reinink / Vanstiphout, 91-108.

Campbell, Jackson J. „,To Hell and Back: Latin Tradition and Literary Use of the Descensus ad inferos", Viator 13 (1982), 107-158.

Cárcel Ortí, María Milagros / Boscá Codina, José Vicente, Visitas pastorales de Valencia (Siglos XIV-XV), Valencia 1996.

Cardelle de Hartmann, Carmen, „Der Dyalogus Agnetis des Gutolf von Heiligenkreuz“, in: Díaz y Díaz / Díaz de Bustamante, 425-435.

_, „Diálogo literario y polémica religiosa en la Edad Media (900-1400)“, in: Alberte González, Antonio / Macías Villalobos, Cristóbal (Hgg.), Actas del Congreso Internacional Cristianismo y tradición latina. Málaga, 25 a 28 de abril de 2000, Málaga 2001, 103-123.

_ , „Die Allgegenwart des Dialogs“ (Rezension über Henkel / Jones / Palmer), in: IASLonline [27.03.2004], URL: <http://iasl.uni-muenchen.de/rezensio/liste/CardelledeHartmann3484640235_835.html>

_ , „Die Processus Sathanae und die Tradition der Satansprozesse“, Mittellateinisches Fahrbuch 40 (2005), 417-430. 
, „El desafio de la literatura latina bajomedieval“, Euphrosyne 31 (2003), 475-482.

„Problemfeld Renaissance-Dialog“, (Rezension über: Guthmüller / Müller, in: IASLonline [14.07.2005], URL: <http://iasl.uni-muenchen.de/rezensio/liste/ CardelledeHartmann3447050861_1201.html>

_ , „Satan vor Gericht. Die ,Processus Satanae“ als Inszenierung juristischer Rhetorik“, in: Kofler, Wolfgang / Töchterle, Karlheinz (Hgg.), Pontes III. Die antike Rhetorik in der europäischen Geistesgeschichte, Innsbruck 2005, 191-202.

Carnicelli, Thomas A., King Alfred's Version of St. Augustine's Soliloquies, Cambridge (Massachusetts) 1969.

Carozza, Davy A. I Shey, H. James, Petrarch's Secretum with introduction, notes and critical anthology, New York u. a. 1989.

Casey, Robert P. / Thomson, Robert W., „A Dialogue between Christ and the Devil“, The Fournal of Theological Studies NS 6 (1955), 49-65.

Casper, Bernhard / Kruttschnitt, Elke / Beinert, Wolfgang / Hunold, Gerfried / Stadler, Anton Paul / Spendel, Stefanie, Art. „Dialog, Dialogik“, Lexikon fir Theologie und Kirche, Bd. 3 (1995), 191-196.

Cataudella, Quintino, „I Soliloqui di Agostino e il libro delle Tusculane“, Aevum 40 (1966), 550-552.

Cenci, Cesare, Manoscritti francescani della BN di Napoli, Bd. 1, Quaracchi 1971.

Cenni, Annamaria, Art. „Auxilius“, in: Chiesa / Castaldi, TE.TRA.2, 129-132.

- , „Eugenius Vulgarius“, in: Chiesa / Castaldi, TE.TRA.2, 174-180.

Chaparro Gómez, César, „Isidoro de Sevilla y los géneros literarios“, Excerpta philologica 1, 1 (1991), 175-188.

— , „Observaciones sobre el título y capitulación del libro sexto de las ,Etimologías de Isidoro de Sevilla“, Anuario de estudios filológicos 8 (1985), 61-66.

Châtillon, Jean, Art. „Prière. III. La tradition chrétienne. C. Prière au Moyen Âge“, DictSpir 12, 2 (1986), 227 1-2288.

Chazan, Robert, Medieval Stereotypes and Modern Antisemitism, Berkeley/Los Angeles 1997.

Chiecchi, Giuseppe, La parola del dolore. Primi studi sulla letteratura consolatoria tra Medioevo e Umanesimo, Padova 2005.

Chiesa, Paolo / Castaldi, Lucia (Hgg.), La trasmissione dei testi latini del Medioevo. Mediaeval Latin Texts and their Transmission. TE.TRA.1, Firenze 2004.

_- La trasmissione dei testi latini del Medioevo. Mediaeval Latin Texts and their Transmission. TE.TRA.2, Firenze 2005.

Gizek, Alexandru, Imitatio et tractatio. Die literarisch-rhetorischen Grundlagen der Nachahmung in Antike und Mittelalter, Tübingen 1994.

__ , ,Zur literarischen und rhetorischen Bestimmung der Schrift Collatio Alexandri Magnis, regis Macedonum, et Dindimi, regis Bragmanorum, de philosophia per litteras facta", Rhetorica 4 (1986), 111-136.

Clark, Francis, The Gregorian Dialogues and the Origins of Benedictine Monasticism, Leiden / Boston 2003.

- The Pseudo-Gregorian Dialogues, Leiden 1987.

Codoñer Merino, Carmen, „Evolución de la lexicografia latina medieval“, in: Pérez González, Maurilio (Hg.), Actas del II Congreso Hispánico de Latín Medieval (León, 11-14 de Noviembre de 1997), Bd. 1, León 1998, 39-50.

— , „Isidorus Hispalensis ep. 1. Etymologiae“, in: Chiesa / Castaldi, TE.TRA.2, 274-299.

Cohen, Jeremy, The Friars and the Fews. The Evolution of Medieval Anti-fudaism, Ithaca / London 1982.

Comunicazione e ricezione del documento cristiano in epoca tardoantica. XXXII Incontro di studiosi dell'antichità cristiana. Roma, 8-10 maggio 2003, Roma 2004.

Constable, Giles, The Reformation of the Twelfth Century, Cambridge 1996. 
Cottier, Jean-François, Anima mea: Prières privées et textes de dévotion du Moyen Age latin, Turnhout 2003.

Courcelle, Pierre, La Consolation de Philosophie dans la tradition littéraire. Antécédents et postérité de Boèce, Paris 1967.

_ Les Confessions de Saint Augustin dans la tradition littéraire. Antécédents et postérité, Paris 1963.

Courcelles, Dominique de, La parole risquée de Raymond Lulle. Entre le judaisme, le christianisme et l'islam, Paris 1993.

Crisciani, Chiara, „Esperienza, communicazione e scrittura in alchimia (secoli XIIIXIV)", in: Galuzzi, Massimo / Micheli, Gianni / Monti, Maria Teresa (Hgg.), Le forme della comunicazione scientifica, Milano 1998, 85-110.

—_, „La Quaestio de alchimia fra ,200 e ,300“, Medioevo 2 (1976), 119-168.

Crisciani, Chiara / Pereira, Michela, L'arte del sole e della luna. Alchimia e Filosofia nel Medioevo, Spoleto 1996.

Cupaiuolo, Giovanni, Introduzione al De ira di Seneca, Napoli 1975.

Curschmann, Michael, Art. „Dialogus Salomonis et Marcolfi“, VL 2 (1978), 80-86.

-, „Marcolfus deutsch. Mit einem Faksimile des Prosa-Drucks von M. Ayrer (1487)“, in: Haug, Walter / Wachinger, Burghart (Hgg.), Kleinere Erzählformen des 15. und 16. Fahrhunderts, Tübingen 1993, 151-255.

Dahan, Gilbert, La polémique chrétienne contre le Fudaïsme au Moyen Âge, Paris 1991.

—, (Hg.), Le brûlement du Talmud à Paris 1242-44, París 1999.

_- Les intellectuels chrétiens et les juifs au Moyen Âge, Paris 1990.

- „Les traductions latines de Thibaud de Sézanne“, in: Dahan, Le brûlement, 95-120.

—- L'exégèse chrétienne de la Bible en Occident médiéval. XII ${ }^{e}-X I V^{e}$ siècle, Paris 1999.

_- ,Saint Anselme, les Juifs, le Judaïsme“, in: Les mutations socio-culturelles au tournant des XI ${ }^{e}-X I I^{e}$ siècles. Etudes Anselmiennes (IV ${ }^{e}$ session), Paris 1984, 521-534.

Daiber, Hans, „Raimundus Lullus in der Auseinandersetzung mit dem Islam. Eine philosophiegeschichtliche Analyse des Liber disputationis Raimundi Christiani et Homeri Saraceni", in: Lutz-Bachmann / Fidora, 136-172.

D‘Alverny, Marie-Thérèse, Alain de Lille. Textes inédits avec une introduction sur sa vie et ses euvres, Paris 1965.

Daly, Lloyd William, ,The Altercatio Hadriani Augusti et Epicteti Philosophi and the Question-and-Answer Dialogue“, in: Daly, Lloyd William / Suchier, Walther, Altercatio Hadriani Augusti et Epicteti Philosophi, Urbana 1939, 5-94.

Dammann, Günter, „Textsorten und literarische Gattungen“, in: Brinker et al. 2000, 546-561.

Dawson, James D., „Richard FitzRalph and the Fourteenth-Gentury Poverty Controversies“, Fournal of Ecclesiastical History 34 (1983), 315-344.

Dazzi, Manlio, Il Mussato preumanista (1261-1329). L'ambiente e l'opera, Vicenza 1964.

De Ghellinck, Joseph, Littérature latine au Moyen Âge, Paris 1939.

De Robertis, Teresa / Resta, Gianvito (Hgg.), Seneca: una vicenda testuale, Firenze 2004.

De Vogüé, Adalbert, Histoire littéraire du mouvement monastique dans l'Antiquité. Première partie: le monachisme latin, (bisher 9 Bde.), Paris 1991-2005.

Delhaye, Philippe, Art. „Florilèges spirituels. II. Florilèges médiévaux d'étique“, DictSpir 5 (1964), 460-475.

Deutinger, Roman, Rahewin von Freising. Ein Gelehrter des 12. Fahrhunderts, Hannover 1999.

Díaz y Díaz, Manuel C. / Díaz de Bustamante, José M. (Hgg.), Poesía Latina Medieval (siglos V-XV). Actas del IV Congreso del „Internationales Mittellateinerkomitee". Santiago de Compostela, 12-15 de septiembre de 2002, Firenze 2005.

Dicke, Gerd, „Aus der Seele gesprochen. Zur Semantik und Pragmatik der Gottesdialoge im ,Fließenden Licht der Gottheit ' Mechthilds von Magdeburg“, in: Henkel / Jones / Palmer, 267-278. 
Diekstra, Frans N. M., A Dialogue between Reason and Adversity, Assen 1968.

Dionisotti, Anna Carlotta, „Greek Grammars and Dictionnaries in Carolingian Europe“, in: Herren, Michael (Hg.), The Sacred Nectar of the Greeks: The Study of Greek in the West in the Early Middle Ages, unter Mitarbeit von Shirley Ann Brown, London 1988, 1-56.

Doignon, Jean, „État des questions relatives aux premiers Dialogues de saint Augustin“, in: Mayer / Chelius, 47-86.

Dolbeau, François, „Critique d'attribution, critique d'authenticité. Réflexions préliminaires", Filologia mediolatina 6-7 (1999-2000), 33-61.

Domínguez, Fernando, „Der Religionsdialog bei Raimundus Lullus. Apologetische Prämissen und kontemplative Grundlage“, in: Jacobi, Gespräche lesen, 263-290.

Douie, Decima L., The Conflict between the Seculars and the Mendicants at the University of Paris in the Thirteenth Century, London 1954.

Drews, Wolfram, „Dogmatischer oder emergenter Dialog? Überlegungen zur Konzeptualisierung theologischer und philosophischer Erkenntnis im Hochmittelalter", Mittellateinisches Fahrbuch 39 (2004), 371-388.

Dronke, Peter, „Laments of the Maries: From the Beginnings to the Mistery Plays“, in: Dronke, Peter, Intellectuals and Poets in Medieval Europe, Roma 1992, 457-489 (Erstveröffentlichung in: Weber, G., Idee - Gestalt - Geschichte, 89-116).

- Verse with prose from Petronius to Dante: the art and scope of the mixed form, Cambridge (Ma.) 1994.

Dufner, Georg, Die Dialoge Gregors des Großen im Wandel der Zeiten und Sprachen, Padova 1968.

Dümmler, Ernst, Auxilius und Vulgarius. Quellen und Forschungen zur Geschichte des Papsthums im Anfange des zehnten Fahrhunderts, Leipzig 1866.

Elfassi, Jacques, „Los centones de los Synonyma de Isidoro de Sevilla“, in: Nascimento, Aires A. / Alberto, Paulo F. (Hgg.), IV Congresso Internacional de Latim Medieval Hispânico. Lisboa, 12-15 de Outubro de 2005. Actas, Lisboa 2006, 393-402.

- Art. „Isidorus Hispalensis ep. 4. Synonyma“, in: Chiesa / Castaldi, TE.tra.1, 218-226.

Emerson, Caryl (Hg.), Critical Essays on Mikhail Bakhtin, New York 1999.

Enders, Markus, „Von der Wahrheits- zur Weisheitssuche im Dialog. Anmerkungen zur Entwicklung der Form des Dialogs im Werk Heinrich Seuses“, in: Jacobi, Gespräche lesen, 359-378.

Enenkel, Karl A. E., „Lucilius redivivus. Zur Seneca-Rezeption des Frühhumanismus: Lombardo della Setas Briefdialog De dispositione vite sue", in: Leonardi, Claudio (Hg.), Gli umanesimi medievali. Atti del II Congresso dell' „Internationales Mittellateinerkomitee“ (Firenze, Certosa del Galluzzo 11-15 settembre 1993), Firenze 1998, 111-120.

Erler, Adalbert, Das Straßburger Münster im Rechtsleben des Mittelalters, Frankfurt a. M. 1954.

Erzgräber, Willi (Hg.), Kontinuität und Transformation der Antike im Mittelalter. Veröffentlichung der Kongreßakten zum Freiburger Symposion des Mediävistenverbandes, Sigmaringen 1989.

Esnos, Geneviève, „Les traductions médiévales françaises et italiennes des Soliloques attribués à Saint Augustin“, Mélanges d'Archéologie et d'Histoire 79 (1967), 299-370.

Farrell, Thomas J., (Hg.) Bakhtin and Medieval Voices, Gainesville (Florida) 1996.

Felten, Franz J. / Jaspert, Nikolas (unter Mitarbeit von Stephanie Haarländer), Vita Religiosa im Mittelalter. Festschrift fuir Kaspar Elm zum 70. Geburtstag, Berlin 1999.

Flint, Valerie, „Heinricus of Augsburg and Honorius Augustodunensis: are they the same person?", Revue bénédictine 92 (1982), 148-158.

Föcking, Marc, „Dyalogum quendam: Petrarcas Secretum und die Arbeit am Dialog im Trecento", in: Hempfer, Möglichkeiten, 75-114.

Foley, Michael P., „Cicero, Augustine, and the Philosophical Roots of the Cassiciacum Dialogues“, Revue des Études Augustiniennes 45 (1999), 51-77.

Fontaine, Jacques, „Isidore de Séville auteur ,ascétique‘: les énigmes des Synonyma“, Studi medievali 6, 1 (1965), 163-195. 
, Isidore de Séville et la culture classique dans l'Espagne wisigothique, 2. überarb. Auflage, Paris 1983.

— , „Le genre littéraire du dialogue monastique dans l'Occident latin des $\mathrm{V}^{\mathrm{e}}$ et $\mathrm{VI}^{\mathrm{e}}$ siècles", in: Starowicyski, Marek (Hg.), The Spirituality of Ancient Monasticism. Acts of the International Colloquium Cracow-Tyniec, 16-19.11.1994, Krakau 1995, 227-250.

Förster, Max, „Das älteste mittellateinische Gesprächsbüchlein“, Romanische Forschungen 27, 2 (1910), 342-348.

Franchini, Enzo, Los debates literarios en la Edad Media, Madrid 2001.

Frank, Barbara / Haye, Thomas / Tophinke, Doris (Hgg.), Gattungen mittelalterlicher Schriftlichkeit, Tübingen 1997.

Frank, Karl Suso, „Fiktive Mündlichkeit als Grundstruktur der monastischen Literatur", in: Kasper / Schreiner, 51-74 (Erstveröffentlichung: Studia Patristica 25 (1993), 356-375).

Friedlein, Roger, Der Dialog bei Ramon Llull. Literarische Gestaltung als apologetische Strategie, Tübingen 2004.

_, „El diàleg en el lul-lisme ibèric medieval, una proposta de sistematització“, in: Arnau Segarra, Pilar / Bover i Font, August (Hgg.), La literatura i l'art en el seu context social, Barcelona 2003, 105-119.

_, „Geleit auf dem Weg zur Wahrheit. Dialoge im Duecento“, in: Hempfer, Möglichkeiten, 39-73.

Friedlein, Roger / Traninger, Anita, Art. „Lullismus“, Historisches Wörterbuch der Rhetorik 5 (2001), 654-661.

Fries, Thomas, „Dialog: Begriff und Geschichte der Gattung“, in: Goetschel, Willi (Hg.), Perspektiven der Dialogik. Zürcher Kolloquium zum 80. Geburtstag von Hermann Levin Goldschmidt, Wien 1994, 29-48.

Fritz, Gerd / Hundsnurscher, Franz (Hgg.), Handbuch der Dialoganalyse, Tübingen 1994.

Fuchs, Franz / Märtl, Claudia, „Ein neuer Text zur Auseinandersetzung zwischen Säkular- und Regularkanonikern im 12. Jahrhundert“, in: Mordek, Hubert (Hg.), Papsttum, Kirche und Recht im Mittelalter: Festschrift für Horst Fuhrmann zum 65. Geburtstag, Tübingen 1991, 277-302.

Fuhrer, Therese, „Augustin: un homme du dialogue“, in: Fux, Pierre-Yves / Roessli, Jean-Michel / Wermelinger, Otto (Hgg.), Augustinus Afer. Saint Augustin: africanité et universalité. Actes du colloque international Alger-Annaba, 1-7 avril 2001, Fribourg 2003, 183-191.

—- Art. „Disputatio“, Augustinus-Lexikon 2 (1996-2002), 503-508.

Funkenstein, Amos, „Basic Types of Christian Anti-Jewish Polemics in the Later Middle Ages“, Viator 2 (1971), 373-382.

Gargan, Luciano, „Per la biblioteca di Giovanni Conversini“, in: Avesani, Rino / Ferrari, Mirella / Foffano, Tino / Frasso, Giuseppe / Sottili, Agostino (Hgg.), Vestigia. Studi in onore di Giuseppe Billanovich, Bd. 1, Roma 1984, 365-385.

Gärtner, Kurt, Art. „Vita beatae virginis Mariae et salvatoris rhythmica“, VL 10 (1999), 436-443.

Gauss, Julia, „Die Auseinandersetzung mit Judentum und Islam bei Anselm“, in: Kohlenberger, Helmut (Hg.), Die Wirkungsgeschichte Anselms von Canterbury, Bd. 2, Analecta Anselmiana 4, 2, Frankfurt a. M. 1975, 101-110.

Geiß, Jürgen, Zentren der Petrarca-Rezeption in Deutschland (um 1470-1525). Rezeptionsgeschichtliche Studien und Katalog der lateinischen Drucküberlieferung, Wiesbaden 2002.

Giordano, Carmela, ,,Die Elucidarium-Rezeption in den germanischen Literaturen des Mittelalters. Ein Überblick“, Mittellateinisches Fahrbuch 38, 1 (2003), 171-187.

Glaser, Hubert, „Wilhelm von Saint-Denis. Ein Humanist aus der Umgebung des Abtes Suger und die Krise seiner Abtei von 1151 bis 1153“, Historisches Fahrbuch 85 (1965), 257-322.

Godding, Robert, „Tra due anniversari: Gregorio Magno alla luce degli studi recenti (1991-2003)", in: Gregorio Magno nel XIV centenario della morte (Roma, 22-25 ottobre 2003), Roma 2004, 89-106. 
Goering, Joseph, William de Montibus (c. 1140-1213). The Schools and the Literature of Pastoral Care, Toronto 1992.

Görgemanns, Herwig, Art. „Dialog“, Der neue Pauly. Enzyklopädie der Antike, Bd. 3 (1997), 517-521.

Gottschall, Dagmar, Das Elucidarium des Honorius Augustodunensis. Untersuchungen zu seiner Überlieferungs- und Rezeptionsgeschichte im deutschsprachigen Raum mit Ausgabe der niederdeutschen Übersetzung, Tübingen 1992.

Graham, Angus A., „Who read Albertanus? Insight from the manuscript transmission“, in: Spinelli, Albertano da Brescia, 69-82.

Grégoire, Reginald, Art. „Sermo“, Dizionario patristico e di antichità cristiane, Bd. 2 (1984), 3157-3163.

Griese, Sabine, Salomon und Markolf. Ein literarischer Komplex im Mittelalter und in der frühen Neuzeit, Tübingen 1999.

Gröber, Gustav, Übersicht über die lateinische Literatur von der Mitte des VI. Fahrhunderts bis zur Mitte des XIV. Fahrhunderts, Straßburg 1902, ND München 1962.

Gruber, Joachim, Art. „Dialog. I. Allgemeines. II. Spätantike. 2. Lateinische Literatur“, LMA 3 (1986), 946-948.

Grubmüller, Klaus, „Gattungskonstitution im Mittelalter“, in: Palmer, Nigel / Schiewer, Hans-Jochen (Hgg.), Mittelalterliche Literatur und Kunst im Spannungsfeld von Hof und Kloster, Tübingen 1999, 193-210.

— Art. „Vocabularius Ex quo“, VL 10 (1999), 469-473.

Gurjewitsch, Aaron J., Mittelalterliche Volkskultur, deutsche Übers. von Matthias Springer, 2. Auflage, München 1992 (russisch 1981).

Guthmüller, Bodo / Müller, Wolfgang G. (Hgg.), Dialog und Gesprächskultur in der Renaissance, Wiesbaden 2004.

Haacke, Rhaban, „Die Überlieferung der Schriften Ruperts von Deutz“, Deutsches Archiv 16 (1960), 397-436.

__ , „Nachlese zur Überlieferung der Schriften Ruperts von Deutz“, Deutsches Archiv 26 (1970), 528-540.

Hadot, Ilsetraut, Seneca und die griechisch-römische Tradition der Seelenleitung, Berlin 1969.

Hadot, Pierre, „Exercices spirituels antiques et ,philosophie chrétienne“", in: Hadot, Pierre, Exercices spirituels et philosophie antique, 2. erw. Auflage Paris 2002 (1. Ed. Paris 1993), 75-98.

_ - „La préhistoire des genres littéraires philosophiques médiévaux dans l'Antiquitée, in: Les genres littéraires, $1-9$.

Halleux, Roger, Les textes alchimiques, Typologie des sources du Moyen Âge occidental 32, Turnhout 1979.

Halsall, Guy (Hg.), Humour, History and Politics in Late Antiquity and the Early Middle Ages, Cambridge 2002.

Hamesse, Jacqueline, „Les florilèges philosophiques du XIII ${ }^{\mathrm{e}}$ au $\mathrm{XV}^{\mathrm{e}}$ siècle“, in: Les genres littéraires, 181-191.

Harmening, Dieter, Superstitio. Überlieferungs- und theoriegeschichtliche Untersuchungen zur kirchlich-theologischen Aberglaubenliteratur des Mittelalters, Berlin 1979.

Häsner, Bernd, „Der Dialog: Strukturelemente einer Gattung zwischen Fiktion und Theoriebildung", in: Hempfer, Poetik, 13-65.

Haug, Walter, „Der Ackermann und der Tod“, in: Stierle / Warning, Das Gespräch, 281-286.

Haverkamp, Alfred (Hg.), Fuden und Christen zur Zeit der Kreuzzüge, Sigmaringen 1999.

Haye, Thomas, Das Lehrgedicht im Mittelalter. Analyse einer Gattung, Leiden u. a. 1997.

Hehle, Christine, Boethius in St. Gallen. Die Bearbeitung der "Consolatio Philosophiae" durch Notker Teutonicus zwischen Tradition und Innovation, Tübingen 2002.

Heimann, Claudia, Nicolaus Eymerich (vor 1320-1399) - praedicator veridicus, inquisitor intrepidus, doctor egregius. Leben und Werk eines Inquisitors, Münster 2001. 
Heinemann, Wolfgang, „Aspekte der Textsortendifferenzierung“, in: Brinker u. a., $523-546$.

- ,Textsorte - Textmuster - Texttyp“, in: Brinker u. a., 507-523.

Heitmann, Klaus, „Augustins Lehre in Petrarcas Secretum“, Bibliothèque d'Humanisme et Renaissance 12 (1960), 34-53. Auch in: Buck, 282-307. Italienische Übersetzung: „L'insegnamento agostiniano nel Secretum del Petrarca“, in: Petrarca e il Petrarchismo, Bologna 1961, 187-193.

- Fortuna und Virtus. Eine Studie zu Petrarcas Lebensweisheit, Köln-Graz 1958.

_, „La genesi del De remediis utriusque fortunae del Petrarca“, Convivium NF 25, 1 (1957), 9-30.

Hempfer, Klaus W., „Einleitung“, in: ders., Poetik, 7-12.

—, Gattungstheorie. Information und Synthese, München 1973.

-, „Lektüren von Dialogen“, in: ders., Möglichkeiten, 1-38.

- (Hg.), Möglichkeiten des Dialogs: Struktur und Funktion einer literarischen Gattung, Stuttgart 2002.

- (Hg.), Poetik des Dialogs. Aktuelle Theorie und rinascimentales Selbstverständnis, Stuttgart 2004.

- „Sinnrelationen zwischen Texten. Petrarcas Secretum und Canzoniere“, GermanischRomanische Monatsschrift 45 (1995), 156-176.

, , „Vorwort“, in: ders., Möglichkeiten, VII-XV.

Henkel, Nikolaus, Deutsche Übersetzungen lateinischer Schultexte. Ihre Verbreitung und Funktion im Mittelalter und in der frühen Neuzeit, München 1988.

Henkel, Nikolaus / Jones, Martin H. / Palmer, Nigel F. (Hgg.): Dialoge. Sprachliche Kommunikation in und zwischen Texten im deutschen Mittelalter. Hamburger Colloquium 1999, Tübingen 2003.

Herbers, Klaus, Art. „Formosus, Papst“, LMA 4 (1989), 655-656.

Herrmann, Erwin, „Der Fürstenspiegel des Michael von Prag“, Historisches Fahrbuch 91 (1971), 22-45.

Hess-Lüttich, Ernest W. B., Art. „Dialog“, Historisches Wörterbuch der Rhetorik 2 (1994), 606-621.

— , „Gesprächsanalyse in der Literaturwissenschaft“, in: Brinker u. a., 1640-1655.

— , „Gesprächsformen in der Literatur“, in: Brinker u. a., 1619-1632.

Hill, Joyce, „Learning Latin in Anglo-Saxon England: Traditions, Texts and Techniques", in: Jones, Sarah Rees, Learning and literacy in Medieval England and abroad, Turnhout 2003, 7-29.

Hillgarth, Jocelyn N., „,The Disputation of Majorca (1286): two new editions“, Euphrosyne 22 (1994) - In honorem Prof. Manuel C. Díaz y Díaz, 403-413.

Hilsenbeck, Renate, Lehrdialog und Laienunterweisung. Der deutsche „Lucidarius“ im Kontext lateinischer Dialogtradition, Diss. Eichstätt 1990 (Mikrofiche).

Hirzel, Rudolf, Der Dialog. Ein literarhistorischer Versuch, Bd. 1 u. 2, Leipzig 1895.

Hödl, Ludwig, Art. „Quaestio, Quaestionenliteratur“, LMA 7 (1995), 349-350.

Hoffmann, Manfred, Der Dialog bei den christlichen Schriftstellern der ersten vier Jahrhunderte, Berlin 1966.

Holtz, Louis, Donat et la tradition de l'enseignement grammatical. Étude sur l'Ars Donati et sa diffusion (IV $V^{e}-I X^{e}$ siècle) et édition critique, Paris 1981.

Honemann, Volker, „Formen symbolischer Kommunikation in deutscher Literatur des Mittelalters", in: Röckelein, Hedwig (Hg.), Kommunikation = Das Mittelalter 6, 1 (2001), 19-30.

Honnacker, Hans, Der literarische Dialog des Primo Cinquecento. Inszenierungsstrategien und ,Spielraum', Baden-Baden 2002.

Hopkins, Jasper, „Anselm's Debate with Gaunilo“, in: Kohlenberger, Helmut (Hg.), Saint Anselme, ses Précurseurs et ses Contemporains, Analecta Anselmiana 5, Frankfurt a. M. 1976, 25-53. 
Horst, Ulrich, Evangelische Armut und Kirche. Thomas von Aquin und die Armutskontroversen des 13. und beginnenden 14. Fahrhunderts, Berlin 1992.

Hösle, Vittorio, Der philosophische Dialog, München 2006.

Höver, Werner, Art. ,,Johann von Neumarkt“, VL 4 (1982), 686-695.

Hudson, Anne, The Premature Reformation. Wycliffite Texts and Lollard History, Oxford 1988.

Hudson, John, „Administration, Family and Perceptions of the Past in Late TwelfthCentury England: Richard FitzNigel and the Dialogue of the Exchequer", in: Magdalino, Paul (Hg.), The Perception of the Past in Twelfth-Century Europe, London / Rio Grande (Ohio) 1992, 75-98.

Huglo, Michel, Les Tonaires. Inventaire, Analyse, Comparaison, Paris 1971.

, „Un nouveau manuscrit du Dialogue sur la musique du pseudo-Odon (Troyes, Bibliothèque Municipale 2142)“, Revue d'histoire des textes 9 (1979), 299-314.

Huizinga, Johan, Homo ludens. Vom Ursprung der Kultur im Spiel, dt. Übers. von H. Nachod, 2. mit einem Nachwort von Andreas Flitner ergänzten Ausgabe, Hamburg 1997 (niederländ. Original Homo ludens erschienen 1938).

Hunt, Richard W., ,,The Disputation of Peter of Cornwall against Symon the Jew“, in: Hunt, Richard W. / Pantin, William A. / Southern, Richard W. (Hgg.), Studies in Medieval History presented to Frederick Maurice Powicke, Oxford 1948, 143-156.

Hüpper, Dagmar, „Buoh und schrift. Gattungen und Textsorten in frühmittelalterlichen volkssprachigen Schriftzeugnissen. Zur Ausbildung einer Begrifflichkeit“, Frühmittelalterliche Studien 20 (1986), 93-122.

Iadanza, Mario, „Il tema della paternità gregoriana dei Dialogi en la tradizione manoscritta nei secoli VII e VIII“", Benedictina 42 (1995), 315-334.

Ianucci, Amilcare, „The Gospel of Nicodemus in Medieval Italian Literature: A Preliminary Assessment", in: Izydorczyk, The Medieval Gospel, 165-205.

Illmer, Detlef, Formen der Erziehung und Wissensvermittlung im frühen Mittelalter, München 1971.

Imbach, Ruedi, „Lulle face aux Averroïstes parisiens“, in: Raymond Lulle et le Pays d'Oc (Cahiers de Fanjeaux 22), Toulouse 1987, 261-282.

Inguanez, Maurus, Catalogi codicum Casinensium antiqui (saec. VIII-XV), Montecassino 1941.

Izydorczyk, Zbigniew, Manuscripts of the Evangelium Nicodemi: a census, Toronto 1993.

— , ,The Evangelium Nicodemi in the Latin Middle Ages“, in: Izydorczyk, The Medieval Gospel, 43-101.

(Hg.), The Medieval Gospel of Nicodemus. Texts, Intertexts, and Contexts in Western Europe, Tempe (Arizona) 1997.

Jacob, Christian, „Questions sur les questions: Archéologie d'une pratique intellectuelle et d'une forme discursive", in: Volgers / Zamagni, 25-54.

Jacobi, Klaus, „Alkuin - Dialoge aus dem Schulunterricht“, in: Jacobi, Gespräche lesen, 55-61.

—_, „Dialogus Ratii et Everardi. Gründliche Gedanken unterhaltsam in Gesprächsform gebracht", in: Jacobi, Gespräche lesen, 243-263.

—_, „Einleitung“", in: Jacobi, Gespräche lesen, 9-22.

(Hg.), Gespräche lesen. Philosophische Dialoge im Mittelalter, Tübingen 1999.

Janota, Johannes, „Grundriss zu einer Geschichte der deutschen Literatur im Spätmittelalter 1220/30-1500/20“, Beiträge zur Geschichte der deutschen Sprache und Literatur 123 (2001), 397-427.

Jeudy, Colette, Marsile de Padoue. Evres mineures, Paris 1979.

Jones, David, An early witness to the nature of the canonical order in the twelfth century. A study in the life and writings of Adam Scot, with particular reference to his understanding of the rule of St. Augustine, Analecta Cartusiana 151, Salzburg 1999.

Jotischky, Andrew, The Carmelites and Antiquity. Mendicants and their Pasts in the Middle Ages, Oxford 2002. 
Kadlec, Jaroslav, Art. „Nikolaus von Jauer“, VL 6 (1987), 1078-1081.

Kampe, Jürgen, Problem „Reformationsdialog“. Untersuchungen zu einer Gattung im reformatorischen Medienwettstreit, Tübingen 1997.

Kantorowicz, Hermann, Studies in the Glossators of the Roman Law. Newly discovered writings of the twelfth century, unter Mitarbeit von William Warwick Buckland, Cambridge 1938, (ND ergänzt und korrigiert von Peter Weimar, Aalen 1969).

Karnein, Alfred, „Petrarca in Deutschland. Zur Rezeption seiner lateinischen Werke im 15. und 16. Jahrhundert", in: Weber, G., Idee - Gestalt - Geschichte, 159-186.

Kasper, Clemens, Art. „Salonius“, Lexikon der antiken christlichen Literatur, 3. vollst. neu bearb. und erw. Auflage, Freiburg u. a. 2002, 620.

Kasper, Clemens M. / Schreiner, Klaus (Hgg.), Viva vox und ratio scripta: Mündliche und schriftliche Kommunikationsformen im Mönchtum des Mittelalters, Münster 1997.

Kasten, Ingrid, „Bachtin und der höfische Roman“, in: Lindemann, Dorothee / Volkmann, Berndt / Wegera, Klaus-Peter (Hgg.), bickelwort und wildiu maere. Festschrift fiur Eberhard Nellmann zum 65. Geburtstag, Göppingen 1995, 51-70.

- Studien zu Thematik und Form des mittelhochdeutschen Streitgedichts, Diss. Hamburg 1973.

Kästner, Hannes, Mittelalterliche Lehrgespräche. Textlinguistische Analysen, Studien zur poetischen Funktion und pädagogischen Intention, Berlin 1978.

Kendall, Ritchie D., The Drama of Dissent. The Radical Poetics of Nonconformity, 1380-1590, Chapel Hill (North Carolina) / London 1986.

Kienzler, Klaus, „Proslogion 1: Form und Gestalt“, in: Luscombe / Evans, 38-55.

Kilian, Jörg, Lehrgespräch und Sprachgeschichte. Untersuchungen zur historischen Dialogforschung, Tübingen 2002.

Kindermann, Udo, „Gattungssysteme im Mittelalter“, in: Erzgräber, Kontinuität und Transformation, 303-313.

Kintzinger, Martin, „Viri religiosi et literati. Kleriker am Fürstenhof im späten Mittelalter“, in: Felten / Jaspert, 543-562.

Kleihues, Alexandra, Der Dialog als Form. Analysen zu Shaftesbury, Diderot, Madame d'Épinay und Voltaire, Würzburg 2002.

Klopsch, Paul, Einführung in die Dichtungslehren des lateinischen Mittelalters, Darmstadt 1980.

Knoche, Ulrich, Die römische Satire, 4. Auflage, Göttingen 1982.

Knysh, George, Political Ockhamism, Winnipeg 1996.

Kock, Thomas, Die Buchkultur der Devotio moderna: Handschriftenproduktion, Literaturversorgung und Bibliotheksaufbau im Zeitalter des Medienwechsels, Frankfurt a. M. u. a. 1999.

Koehler, Théodore, Art. „Planctus Mariae“, DictSpir 12, 2 (1986), 1795-1800.

Krause, Wolf-Dieter, „Zum Begriff der Textsorte“, in: Krause, Wolf-Dieter (Hg.), Textsorten. Kommunikationslinguistische und konfrontative Aspekte, Frankfurt a.M. et al., 2000, 11-33.

Kristeller, Paul Oskar, „Das moralische Denken des Renaissance-Humanismus“, in: ders., Humanismus und Renaissance II, 30-84.

__ , „Der Gelehrte und sein Publikum im späten Mittelalter und in der Renaissance“, in: ders., Humanismus und Renaissance II, München 1976, 223-243.

- Latin Manuscript Books before 1600. A List of the Printed Catalogues and Unpublished Inventories of Extant Collections, 4. von Sigrid Krämer erweiterte Auflage, München 1993.

Kristeller, Paul Oskar / Brown, Virginia / Cranz, Ferdinand E. (Hgg.), Catalogus translationum et commentariorum: Mediaeval and Renaissance Latin Translations and Commentaries. Annotated Lists and Guides, bisher 8 Bde., Washington 1960ff.

Külzer, Andreas, Disputationes Graecae contra Iudaeos: Untersuchungen zur byzantinischen antijüdischen Dialogliteratur und ihrem Fudenbild, Stuttgart / Leipzig 1999.

Kunzmann, Peter, „Aelred von Rievaulx: De spiritali amicitia. Ein unfreundlicher Dialog über Freundschaft“, in: Jacobi, Gespräche lesen, 231-242. 
Kushner, Eva, Le dialogue à la Renaissance. Histoire et poétique, Genève 2004.

— , „Le dialogue en France au XVI $\mathrm{X}^{\mathrm{e}}$ siècle: quelques critères“, in: Kushner, Le dialogue à la Renaissance, 39-53 (zuerst erschienen in: Revue canadienne de littérature comparée, printemps 1978, 485-501).

Kuttner, Stephan / Rathbone, Eleanor, „Anglo-Norman Canonists of the Twelfth Century: An Introductory Study“, Traditio 7 (1949-1951), 279-358.

Lachmann, Renate (Hg.), Dialogizität, München 1982.

Lambert, Malcolm D., Povertà francescana, Milano 1995 (2. aktualisierte Auflage von: Franciscan Poverty. The Doctrine of the Absolute Poverty of Christ and the Apostles in the Franciscan Order 1210-1323, London 1961).

Lampe, Geoffrey W. H., A Patristic Greek Lexicon, Oxford 1961.

Lapidge, Michael, ,Three Latin poems from Aethelwold's school at Winchester", AngloSaxon England 1 (1972), 85-123.

Lausberg, Marion, „Senecae operum fragmenta: Überblick und Forschungsbericht“, Aufstieg und Niedergang der römischen Welt II.36.3 (1989), 1879-1961.

Lawn, Brian, The Rise and Decline of the Scholastic Quaestio Disputata. With Special Emphasis on its Use in the Teaching of Medicine and Science, Leiden u. a. 1993.

- The Salernitan Questions. An Introduction to the History of Medieval and Renaissance Problem Literature, Oxford 1963.

Lazzari, Loredana, „I colloquia nelle scuole monastiche anglosassoni tra la fine del $\mathrm{X}^{\mathrm{e}}$ la prima metà dell'XI secolo“, Studi Medievali 3. s., 44 (2003), 147-177.

Leclercq, Jean, „Introduction. I. L'auteur et l'œuvre“, in: Friedlander, Colette / Leclercq, Jean / Raciti, Gaetano, Galand de Reigny. Parabolaire, Sources Chrétiennes 378, Paris 1992, 13-37.

- L'amour des lettres et le désir de Dieu. Initiation aux auteurs monastiques du Moyen Âge, Paris 1957 [deutsche Übersetzung von Johannes und Nicole Stöber: Wissenschaft und Gottverlangen. Zur Mönchstheologie des Mittelalters, Düsseldorf 1963].

__ , „La plus ancienne collection d'œuvres complètes de Saint Bernard“, in: ders., Études sur Saint Bernard et le texte de ses écrits = Analecta sacri ordinis Cisterciensis 9 (1953), 124-136.

Leff, Gordon, Heresy in the Later Middle Ages. The Relation of Heterodoxy to Dissent c. 12501450, 2. Auflage Manchester 1999 (1. Auflage in 2 Bde. Manchester 1967).

Lehmann, Paul, „Aufgaben und Anregungen der lateinischen Philologie des Mittelalters", in: ders., Erforschung des Mittelalters. Ausgewählte Abhandlungen und Aufsätze, Bd. 1, Leipzig 1941, 1-46 (erste Veröffentlichung: Sitzungsberichte der Kgl. Bayerischen Akademie der Wissenschaften. Philosophisch-philologische und historische Klasse, München 1918, 8. Abh.).

—, Die Parodie im Mittelalter, 2. neu bearb. und erw. Auflage, Stuttgart 1963.

Leonardi, Glaudio, Art. „Glaudius von Turin“, LMA 2 (1983), 2132-2133.

- (Hg.), Gli umanesimi medievali. Atti del II Congresso dell' „Internationales Mittellateiner Komitee" (Firenze, Certosa del Galluzzo 11-15 settembre 1993), Firenze 1998.

- u. a., Letteratura latina medievale (secoli VI-XV). Un manuale, Firenze 2002.

Leoncini, Letizia, „Forme editoriali del testo e commento figurato. II. Il caso di Giovanni Conversini da Ravenna“", in: Intorno al testo. Tipologie del corredo esegetico e soluzioni editoriali. Atti del convegno di Urbino 1-3 ottobre 2001, Roma 2003, 485-495.

$\longrightarrow$, „La novella a corte: Giovanni Conversini da Ravenna“, in: Albanese, Gabriella / Battaglia Ricci, Lucia / Bessi, Rossella (Hgg.), Favole, parabole, istorie. Le forme della scrittura novellistica dal Medioevo al Rinascimento. Atti del Convegno di Pisa 26-28 ottobre 1998, Roma 2000, 189-222.

Leppin, Volker, „Die Aufwertung theologischer Laienkompetenz bei Wilhelm von Ockham", in: Strauß, Elisabeth (Hg.), Dilettanten und Wissenschaft. Zur Geschichte und Aktualität eines wechselvollen Verhältnisses, Amsterdam / Atlanta 1996, 35-48.

Lerer, Seth, Boethius and Dialogue. Literary Method in the Consolation of Philosophy, Princeton 1985. 
Les genres littéraires dans les sources théologiques et philosophiques médiévales. Défnition, critique et exploitation. Actes du Colloque international de Louvain-la-Neuve 25-27 mai 1981, Louvainla-Neuve 1982.

Lévy, Carlos, „La conversation à Rome à la fin de la République: des pratiques sans théorie?", Rhetorica 11 (1993), 399-420.

Lewis, Bernhard / Niewöhner, Friedrich (Hgg.), Religionsgespräche im Mittelalter, Wiesbaden 1992.

Liebermann, Felix, Einleitung in den Dialogus de scaccario, Göttingen 1875.

Lüddecke, Dirk, „Veritas exagitata. Überlegungen zu Ockhams politischer Lehre und ihrer Darbietung im Dialogus", in: Jacobi, Gespräche lesen, 323-347.

Ludwig, Walther, „Formen und Bezüge frühneuzeitlicher lateinischer Dialoge“, in: Guthmüller / Müller, 59-103.

Luscombe, David / Evans, Gillian (Hgg.), Anselm: Aosta, Bec and Canterbury, Sheffield 1996.

Lütcke, Karl-Heinrich, Art. „Auctoritas“, Augustinus-Lexikon 1 (1986-1994), 498-510.

Lutz-Bachmann, Matthias / Fidora, Alexander (Hgg.), Juden, Christen und Muslime. Religionsdialoge im Mittelalter, Darmstadt 2004.

Maas, Wilhelm, Gott und die Hölle. Studien zum Descensus Christi, Einsiedeln 1979.

Maccoby, Hyam, Fudaism on Trial. Fereish-Christian Disputations in the Middle Ages, London / Toronto 1982.

Madec, Goulven, „Le néoplatonisme dans la conversion d'Augustin. État d'une question centenaire (depuis Harnack et Boissier, 1888)“, in: Mayer / Chelius, 9-25.

Manitius, Max, Geschichte der lateinischen Literatur des Mittelalters, Bd. 1-3, München 1911-1931.

Mann, Nicholas, „La fortune de Pétrarque en France: Recherches sur le De remediis“, Studi Francesi 39 (1969), 1-15.

— , „Petrarch and the Transmission of Classical Elements“, in: Bolgar, R. R. (Hg.), Classical Influences on European Culture A.D. 500-1500, Cambridge 1971, 217-224.

—, „Recherches sur l'influence et la diffusion du ,De remediis' de Pétrarque aux Pays-Bas", in: Verbeke, Gérard / Ijsewijn, Jozef, The Late Middle Ages and the Dawn of Humanism outside Italy, Leuven 1972, 78-88

Manselli, Raoul, „La polémique contre les juifs dans la polémique antihérétique“, in: Vicaire, / Blumenkranz, fuifs et judaïsme, 251-267.

Mantello, Frank A. C. / Rigg, Arthur G. (Hgg.) Medieval Latin. An Introduction and Bibliographical Guide, Washington D.C. 1996.

Marone, Paola, „Le Quaestiones et Responsiones sulla Bibbia nella letteratura cristiana di IV-VI secolo", in: Comunicazione e ricezione, 43-73.

Marsh, David, Lucian and the Latins. Humor and Humanism in the Early Renaissance, Ann Arbor (Michigan) 1998.

Martin, Josef, Symposion. Die Geschichte einer literarischen Form, Paderborn 1931.

Martinez, Matias, „Dialogizität, Intertextualität, Gedächtnis“, in: Arnold, Heinz Ludwig / Detering, Heinrich (Hgg.), Grundzüge der Literaturwissenschaft, München 1996, 430-445.

Marx, Carl W., „The Gospel of Nicodemus in Old English and Middle English“, in: Izydorczyk, The Medieval Gospel, 207-259.

Matter, E. Ann, „Alcuin's Question-and-Answer Texts“, Rivista di storia della filosofia 45 (1990), 645-656.

Mattioli, Emilio, Luciano e l'Umanesimo, Napoli 1980.

Mayer, Cornelius / Chelius, Karl Heinz (Hgg.), Internationales Symposion über den Stand der Augustinus-Forschung, Würzburg 1989.

McCarthy, T. J. H., ,,The identity of Master Henry of Augsburg (d. 1083)“, Revue bénédictine 114 (2004), 140-157.

McClure, George W., Sorrow and Consolation in Italian Humanism, Princeton 1990.

McGregor, Alexander, „Mussato's Commentary on Seneca's Tragedies. New Fragments", Illinois Classical Studies 5 (1980), 149-162. 
McNally, Robert, „The Pseudo-Isidorian De vetere et novo testamento quaestiones“, Traditio 14 (1963), 37-50.

Meersseman, Gilles Gerard, „Seneca maestro di spiritualità nei suoi opuscoli apocrifi dal XII al XV secolo“, Italia Medioevale e Umanistica 16 (1973), 43-135.

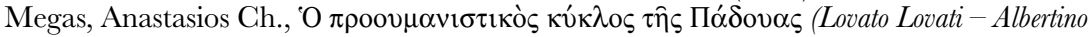

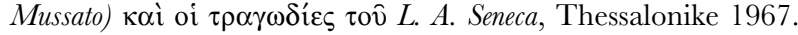

Meier, Christel, „Das Autorbild als Kommunikationsmittel zwischen Text und Leser“, in: Communicare e significare nell'Alto Medioevo, Bd. 1, Settimane di studio della Fondazione Centro Italiano di Studi sull'Alto Medievo 52,1, Spoleto 2005, 499-538.

Mentzel-Reuters, Arno, „Literaturbericht Handschriftenkataloge“, Deutsches Archiv 51 (1995), 169-194; 53 (1997), 179-203; 54 (1998), 583-611; 57 (2001), 583-611; 60 (2004), 201-231.

Meuthen, Erich, „,Der Quellenwandel vom Mittelalter zur Neuzeit und seine Folgen für die Kunst der Publikation“, in: Gall, Lothar / Schieffer, Rudolf (Hgg.), Quelleneditionen und kein Ende?, Beihefte der Historischen Zeitschrift 28, München 1999, 17-36.

Mews, Constant J., „St. Anselm and Roscelin: Some New Texts and their Implications. I. The De incarnatione verbi and the Disputatio inter Christianum et Gentilem", Archives d'histoire doctrinale et littéraire du Moyen Âge 58 (1991), 55-98.

Meyvaert, Paul, „The Authentic Dialogues of Gregory the Great“, Sacris Erudiri 43 (2004), 55-129.

Miethke, Jürgen, „Das Publikum politischer Theorie im 14. Jahrhundert. Zur Einführung", in: ders. (Hg.), Das Publikum politischer Theorie im 14. Jahrhundert, unter Mitarbeit von Arnold Bühler, München 1992, 1-23.

_ _ De potestate papae. Die päpstliche Amtskompetenz im Widerstreit der politischen Theorie von Thomas von Aquin bis Wilhelm von Ockham, Tübingen 2000.

__ , „Marsilius und Ockham. Publikum und Leser ihrer politischen Schriften im späteren Mittelalter", Medioevo. Rivista di storia della filosofia medievale 6 (1980), 543-567. -, Ockhams Weg zur Sozialphilosophie, Berlin 1969.

—_, „Paradiesischer Zustand - Apostolisches Zeitalter - Franziskanische Armut. Religiöses Selbstverständnis, Zeitkritik und Gesellschaftstheorie im 14. Jahrhundert“, in: Felten / Jaspert, 503-532.

, ,Theologenprozesse in der ersten Phase ihrer institutionellen Ausbildung. Die Verfahren gegen Abaelard und Gilbert von Poitiers", Viator 6 (1975), 87-116.

—_, "Verschriftlichte Mönchstheologie und Zensur", in: Kasper / Schreiner, $177-204$

— , „Zur Bedeutung von Ockhams politischer Philosophie für Zeitgenossen und Nachwelt", in: Vossenkuhl, Wilhelm / Schönberger, Rolf, Die Gegenwart Ockhams, Weinheim 1990, 305-324.

Minnis, Alastair J. (Hg.), The Medieval Boethius. Studies in the Vernacular Translations of De Consolatione Philosophiae, Cambridge 1987.

Minnis, Alastair J. / Scott, Alexander B., Medieval Literary Theory and Criticism c. 1100 c. 1375. The Commentary Tradition, 2. Auflage, Oxford 1991.

Mohrmann, Christine, „Praedicare - tractare - sermo“, in: dies., Études sur le latin des chrétiens. II. Latin chrétien et médiéval, Roma 1961, 63-72 (zuerst erschienen in La Maison-Dieu 39 (1954), 97-107).

Mollat du Jourdin, Michel / Vauchez, André, Die Geschichte des Christentums. Religion, Politik, Kultur. Bd. 6. Die Zeit der Zerreißproben (1274-1449), Deutsche Ausgabe bearb. und hrsg. von Bernhard Schimmelpfennig, Freiburg / Basel / Wien 1991 (französische Originalausgabe: Histoire du christianisme des origines à nos jours. Tome VI: Un temps d'épreuves (1274-1449), Paris 1990).

Montclos, Jean de, Lanfranc et Bérenger. La controverse eucharistique du XI siècle, Leuven 1971.

Moores, Elizabeth, „The long and the short of the Contemptus sublimitatis: A Medieval Text in the Making", Manuscripta 34 (1990), 216-232. 
Moos, Peter von, Consolatio. Studien zur mittellateinischen Trostliteratur über den Tod und zum Problem der christlichen Trauer, München 1971.

—_, „Das argumentative Exemplum und die wächserne Nase der Autorität im Mittelalter", in: ders. Rhetorik, 45-67 (zuerst erschienen in: Aerts, Willem J. / Gosman, Martin (Hgg.), Exemplum et similitudo. Alexander the Great and other heroes as points of reference in medieval literature, Groningen 1988, 55-84).

,- , „Die Epistolae duorum amantium und die säkulare Religion der Liebe. Methodenkritische Vorüberlegungen zu einem einmaligen Werk mittellateinischer Briefliteratur", Studi Medievali 44 (2003), 1-115.

—, „Die italienische ars arengandi des 13. Jahrhunderts als Schule der Kommunikation“", in: ders. Rhetorik, 127-152 (zuerst erschienen in: Brunner, Horst / Wolf, Norbert Richard (Hgg.), Wissensliteratur im Mittelalter und in der Frühen Neuzeit. Bedingungen, Typen, Publikum, Sprache, Wiesbaden 1993, 67-90).

_, „Die Kunst der Antwort. Exempla und dicta im lateinischen Mittelalter“, in: ders. Rhetorik, 69-106 (zuerst erschienen in: Haug, Walter / Wachinger, Burghart (Hgg.), Exempel und Exempelsammlungen, Tübingen 1991, 24-58).

—_, Entre histoire et littérature. Communication et culture au Moyen Agge, Firenze 2005.

, Geschichte als Topik. Das rhetorische Exemplum von der Antike zur Neuzeit und die historiae im „Policraticus" Fohanns von Salisbury, 2. Auflage, Hildesheim / Zürich / New York 1996.

— , „Gespräch, Dialogform und Dialog nach älterer Theorie“, in: ders. Rhetorik, 205-228 (zuerst erschienen in: Frank / Haye / Tophinke 1997, 235-259).

-, „L'ars arengandi italienne du XIII ${ }^{\mathrm{e}}$ siècle. Une école de la communication“, in: ders., Entre histoire et littérature, 389-415.

—_ , „Le dialogue latin au Moyen Âge: l'exemple d'Évrard d'Ypres“, in: ders., Entre histoire et littérature, 343-387 (erweiterte Fassung des gleichnamigen Aufsatzes in Annales, économies, sociétés, civilisations, 44.4 (1989), 993-1028).

—_ ,Les Collationes d'Abélard et la ,question juive“ au XII ${ }^{\mathrm{e}}$ siècle“, in: ders., Entre histoire et littérature, 45-86 (aktualisierte Fassung des gleichnamigen Aufsatzes in Fournal des Savants, Juillet-Décembre 1999, 449-489).

__ , „L'exemplum et les exempla des prêcheurs“, in: ders., Entre histoire et littérature, 277-290 (zuerst erschienen in: Berlioz, Jacques / Polo de Beaulieu, Marie-Anne (Hgg.), Les exempla médiévaux: Nouvelles perspectives, Paris 1998, 67-83).

_ _ ,Literatur- und bildungsgeschichtliche Aspekte der Dialogform im lateinischen Mittelalter. Der Dialogus Ratii des Eberhard von Ypern zwischen theologischer disputatio und Scholaren-Komödie“, in: ders. Rhetorik, 3-43 (zuerst erschienen in: Bernt, Günter / Rädle, Fidel / Silagi, Gabriel (Hgg.), Tradition und Wertung. Festschrift fir Franz Brunhölzl zum 65. Geburtstag, Sigmaringen 1989, 165-205).

— , „Rhetorik, Dialektik und civilis scientia im Hochmittelalter“, in: ders. Rhetorik, 239-264 (zuerst erschienen in: Fried, Johannes (Hg.), Dialektik und Rhetorik im früheren und hohen Mittelalter, München 1992, 133-155).

—, Rhetorik, Kommunikation und Medialität. Gesammelte Studien zum Mittelalter, Band II, hg. von Gert Melville, Berlin 2006.

_ , „Sulla retorica dell'exemplum nel Medioevo“, in: Ders., Entre histoire et litterature, 257-276 (zuerst erschienen in: Leonardi, Claudio / Menestò, Enrico (Hgg.), Retorica e poetica tra $i$ secoli XIII e XIV, Firenze 1988, 53-79).

— , ,Zwischen Schriftlichkeit und Mündlichkeit: Dialogische Interaktion im lateinischen Hochmittelalter", Frühmittelalterliche Studien 25 (1991), 300-314.

- Hildebert von Lavardin 1056-1133. Humanitas an der Schwelle des höfischen Zeitalters, Stuttgart 1965.

Moulinier, Laurence, „Unterhaltungen mit dem Teufel: Eine französische Hildegardvita des 15. Jahrhunderts und ihre Quellen“, in: Haverkamp, Alfred (Hg.), Hildegard von Bingen in ihrem historischen Umfeld. Internationaler wissenschaftlicher Kongreß zum 900jährigen Jubiläum, 13.-19. September 1998, Bingen am Rhein, Mainz 2000, 519-547. 
Müller, Hubert, Früher Humanismus in Oberitalien. Albertino Mussato: Ecerinis, Frankfurt a. Main u. a. 1987.

Müller, Wolfgang, „Dialog und Dialogizität in der Renaissance“, in: Guthmüller / Müller, 17-31.

Munk Olsen, Birger, „Les florilèges d'auteurs classiques“, in: Les genres littéraires, 151164.

—_ ,Les florilèges et les abrégés de Sénèque au Moyen Age“, Giornale italiano di Filologia 52 (2000), 163-183.

Nason, Vittore, „Note sulla Dragmalogia de elegibili vite genere di Giovanni Conversini da Ravenna“, Maia. Rivista di letterature classiche 39 (1987), 43-55.

Neumeister, Sebastian, Das Spiel mit der höfischen Liebe. Das altprovenzalische Partimen, München 1969.

Newman, Robert J., „Rediscovering the De remediis fortuitorum“, American Fournal of Philology 109 (1988), 92-107.

Nies, Fritz, „Frage und Antwort als dialogische Struktur im Verhältnis von Autor zu Autor (Werk zu Werk)“, in: Lachmann, 185-189.

Niesner, Manuela, „,Wer mit juden well disputiren“. Deutschsprachige Adversus-fudaeos-Literatur des 14. Jahrhunderts, Tübingen 2005.

Nimmo, Duncan, Reform and Division in the Medieval Franciscan Order. From Saint Francis to the Foundation of the Capuchins, Roma 1987.

— , „The Franciscan Regular Obervance: The Culmination of Medieval Franciscan Reform“, in: Elm, Kaspar (Hg.), Reformbemühungen und Observanzbestrebungen im spätmittelalterlichen Ordenswesen, Berlin 1989, 189-205.

Nothdurft, Klaus-Dieter, Studien zum Einfluss Senecas auf die Philosophie und Theologie des zwölften Fahrhunderts, Leiden-Köln 1963.

O’Daly, Gerald., Art. „Cassiciacum“, Augustinus-Lexikon 1 (1986-1994), 771-781.

- The Poetry of Boethius, Chapel Hill / London 1991.

Oesterreicher, Wulf, „Zur Fundierung von Diskurstraditionen“, in: Frank / Haye / Tophinke, 19-41.

Ohly, Friedrich, „Die Trinität berät über die Erschaffung des Menschen und über seine Erlösung“", Beiträge zur Geschichte der Deutschen Sprache und Literatur 116 (1994), 242-284.

Olphe-Galliard, Michel, Art. „Conférences spirituelles“, DictSpir 2 (1953), 13891405.

Ornato, Ezio, Jean Muret et ses amis Nicolas de Clamanges et Jean de Montreuil. Contribution à l'étude des rapports entre les humanistes de Paris et ceux d'Avignon (1394-1420), Genève Paris 1969.

_ _, „L'umanista Jean Muret ed il suo dialogo De contemptu mortis“, in: Simone, Franco (Hg.), Miscellanea di studi e ricerche sul Quattrocento francese, Torino 1967, 241-355.

Ott, Norbert, Art. „Jacobus de Theramo“, VL 4 (1983), 441-447.

$\longrightarrow$, Rechtspraxis und Heilsgeschichte. Zu Überlieferung, Ikonographie und Gebrauchssituation des deutschen ,Belial', München 1983.

Ourliac, Paul, „Das Schisma und die Konzilien (1378-1449)“, in: Mollat du Jourdin / Vauchez, 75-131.

Pabst, Bernhard, Art. „Prosimetrum“, Historisches Wörterbuch der Rhetorik 7 (2005), 349-355.

_ Prosimetrum. Tradition und Wandel einer Literaturform zwischen Spätantike und Spätmittelalter, 2. Bde., Köln / Weimar / Wien 1994.

Päpke, Max, Das Marienleben des Schweizers Wernher. Mit Nachträgen zu Vögtlins Ausgabe der Vita Marie Rhythmica, Berlin 1913.

Palmer, Nigel F., Art. „Visio Philiberti“, VL 10 (1999), 412-418.

Patschovsky, Alexander, „Der ,Talmudjude‘. Vom mittelalterlichen Ursprung eines neuzeitlichen Themas", in: Haverkamp, Alfred / Ziwes, Franz-Josef (Hgg.), fuden in der christlichen Umwelt während des späten Mittelalters, Berlin 1992, 13-27. 
,Feindbilder der Kirche: Juden und Ketzer im Vergleich (11.-13. Jahrhundert)“, in: Haverkamp, Fuden und Christen, 327-357.

Pedroni, Matteo / Stäuble, Antonio (Hgg.), Il genere ,tenzone“ nelle letterature romanze delle origini, Ravenna 1999.

Perarnau i Espelt, Josep, „El diàleg entre religions en el lul-lisme castellà medieval“, Estudios Lulianos 22 (1978), 241-259.

Pereira, Michela, „Filosofia naturale lulliana e alchimia. Con l'inedito epilogo del Liber de secretis naturae seu de quinta essentia“, Rivista di storia della filosofia 41 (1986), 747-780.

—_ The Alchemical Corpus attributed to Raymond Lull, London 1989.

Perelman, Chaim, „Dialectique et dialogue“, Hermeneutik und Dialektik 2 (1970), 77-83, jetzt auch in: Perelman, Rhétoriques, 9-18.

- „La méthode dialectique el le rôle de l'interlocuteur dans le dialogue“, Rerue de Métaphysique et de Morale 1 (1955), 26-31, jetzt auch in: ders., Rhétoriques, Bruxelles 1989, 53-60.

Perelman, Chaim / Olbrechts-Tyteca, Lucie, La nouvelle rhétorique. Traité de l'argumentation, Paris 1958.

Pérez González, Maurilio, „Poesía satírica en torno a las investiduras: la Altercatio inter Vrbanum et Clementem y el Certamen regis cum papa“, in: Díaz y Díaz / Díaz de Bustamante, 385-398.

Perger, Mischa von, „Deliberativa theoria. Eriugenas dialogische Kunst der Erwägung“, in: Jacobi, Gespräche lesen, 63-99.

—, „Vorläufiges Repertorium philosophischer und theologischer Prosa-Dialoge des lateinischen Mittelalters", in: Jacobi, Gespräche lesen, 435-494.

Perrone, Lorenzo, „Sulla preistoria delle quaestiones nella letteratura patristica. Presupposti e sviluppi del genere litterario fino al IV sec.", Annali di storia dell'esegesi 8,2 (1991), 485-505.

Petersohn, Jürgen, „Apostolus Pomeranorum. Studien zur Geschichte und Bedeutung des Apostelepithetons Bischof Ottos I. von Bamberg“, Historisches Jahrbuch 86 (1966), 257-294.

, „Bemerkungen zu einer neuen Ausgabe der Viten Ottos von Bamberg 2. Herbords Dialog“",Deutsches Archiv zur Erforschung des Mittelalters 33 (1977), 546-569.

Pfister, Manfred, Das Drama: Theorie und Analyse, 11. Aufl., erw. und bibliograf. aktualisierter ND der durchges. und erg. Aufl. 1988, München 2001.

Pflaum, Hiram (später Peri, Hiram), Die religiöse Disputation in der europäischen Dichtung des Mittelalters. I: Der allegorische Streit zwischen Synagoge und Kirche, Genève / Firenze 1935.

Pignatti, Franco, „Il dialogo del Rinascimento. Rassegna della critica“, Giornale storico della letteratura italiana 176 (1999), 408-443.

Pittaluga, Stefano, „Modelli classici e filologia nell'Ecerinis di Albertino Mussato“, Studi medievali 29, 1 (1988), 267-276.

_ - „Seneca tragicus nel X secolo. Eugenio Vulgario e la ricezione provocatoria“, in: ders, La scena interdetta. Teatro e letteratura fra Medioevo e Umanesimo, Napoli 2002, 217-228 (zuerst erschienen in Mittellateinisches Fahrbuch 24-25 (1989-1999) [= Berschin, Walter (Hg.), Lateinische Kultur im X. Fahrhundert. Akten des I. Internationalen Mittellateinerkongresses, Heidelberg 12.-15. Sept. 1988], 383-391).

Platzeck, Erhard, Das Leben des seligen Raimund Lull. Die „Vita coëtanea“ und ausgewählte Texte zum Leben Lulls aus seinen Werken und Zeitdokumenten, Düsseldorf 1964.

Plezia, Marian, „L'histoire dialoguée: procédé d'origine patristique dans l'historiographie médiévale“, Studia Patristica 4 (1961), Texte und Untersuchungen zur Geschichte der Altchristlichen Literatur 79, 490-496.

Poirel, Dominique / Jeudy, Colette / Lescuyer, Matthieu / Szerwiniack, Olivier, „Auteurs et genres littéraires“, in: Vauchez, André / Caby, Cécile (Hgg.), L’Histoire des moines, chanoines et religieux du Moyen Âge. Guide de recherche et documents, Turnhout 2003, 229-289. 
Polizzi, Carlo F., „Nuovi documenti e ricerche sul cenacolo preumanista padovano“, Italia Medioevale e Umanistica 28 (1985), 137-187.

Polock, Marlene, ,„Der Prozeß von 1194 zwischen Orvieto und Savona um das Val di Lago. Mit Edition der Akten und der Bischofsliste von Savona bis zum Ende des 12. Jahrhunderts", Quellen und Forschungen aus italienischen Archiven und Bibliotheken 70 (1990), 46-150.

Pontes, José Maria da Cruz, „Raimundo Lulo e o lulismo português“, Biblos 62 (1986), 51-76.

Poole, Reginald L., The Exchequer in the Twelfth Century, Oxford 1912.

Pourrat, Pierre, „Colloque“, DictSpir 2 (1953), 1123-1130.

Powell, James M., „Albertano da Brescia e i suoi lettori. Studio sulla trasformazione del significato", in: Spinelli, Albertano da Brescia, 83-93.

Preisendanz, Wolfgang, „Zum Beitrag von R. Lachmann, ,Dialogizität und poetische Sprache“", in: Lachmann, 25-28.

Quillen, Carol E., „Petrarch and Augustine“, Fournal of the History of Ideas 53 (1992), 179-207.

- Rereading the Renaissance. Petrarch, Augustine, and the Language of Humanism, Ann Arbor 1998.

Quillet, Jeannine, „Remarques sur l'usage de l'allégorie dans l'œuvre d'A. Mussato“, Medioevo. Rivista di storia della filosofia medievale 11 (1985), 163-172.

Rädle, Fidel, „Literatur gegen Literaturtheorie? Überlegungen zu Gattungsgehorsam und Gattungsverweigerung bei lateinischen Autoren des Mittelalters", in: Frank / Haye / Tophinke, 221-234.

Rapp, Claudia, „Hagiography and monastic literature between Greek East and Latin West in Late Antiquity", in: Cristianità d'Occidente e Cristianità d'Oriente (secoli VI-XI), Bd. 2, Settimane di studio della Fondazione Centro Italiano di Studi sull'Alto Medioevo 51, Spoleto 2004, 1221-1281.

Rawski, Conrad H., „Petrarch's Scholarship in His De remediis utriusque fortune: A Preliminary Inquiry", in: Bernardo, Aldo S. / Levin, Saul (Hgg.), The Classics in the Middle Ages. Papers of the Twentieth Annual Conference of the Center for Medieval and Early Renaissance Studies, Binghamton NY 1990, 283-314.

Reed, Thomas L. Jr., Middle English Debate Poetry and the Aesthetics of Irresolution, Columbia (Missouri) / London 1990.

Reeves, Marjorie, Foachim of Fiore and the Prophetic Future, London 1976.

—. The Influence of Prophecy in the Later Middle Ages. A Study in Foachimism, Oxford 1969 (2. Auflage, Notre Dame 1993).

Regn, Gerhard / Huss, Bernhard, „Pluralisierung von Wahrheit im Individuum: Petrarcas Secretum“, in: dies. (Hgg.), Francesco Petrarca. Secretum meum, Mainz 2004, 493-539.

Reinink, Gerrit J. / Vanstiphout, Herman L. J. (Hgg.), Dispute Poems and Dialogues. Forms and Types of Literary Debates in Semitic and Related Literatures, Leuven 1991.

Reinink, Gerrit J., „Ein syrisches Streitgespräch zwischen Tod und Satan“, in: Reinink / Vanstiphout, 135-152.

Reiss, Edmund, „Conflict and its resolution in Medieval dialogues“, in: Arts libéraux et philosophie au Moyen Âge. Actes du quatrième congrès international de Philosophie médiévale. Montréal 27 août-2 septembre 1967, Montréal / Paris 1969, 863-872.

Rentsch, Thomas, „Die Kultur der quaestio. Zur literarischen Formgeschichte der Philosophie im Mittelalter", in: Gabriel, Gottfried / Schildknecht, Christiane (Hgg.), Literarische Formen der Philosophie, Stuttgart 1990, 73-91.

Reynolds, Leighton D., „The medieval tradition of Seneca's Dialogues“, The Classical Quarterly 62 (NS 18) (1968), 355-372.

Ricklin, Thomas, „Der Dialogus des Petrus Alfonsi. Eine Annäherung““, in: Jacobi, Gespräche lesen, 139-156.

Rico, Francisco, „,Ubi puer, ibi senex‘: Hans Baron y el Secretum de 1353“, in: Il Petrarca 
latino e le origini dell'umanesimo. Atti del Convegno internazionale Firenze 19-22 maggio 1991 = Quaderni Petrarcheschi 9-10 (1992-1993), Bd. 1, 165-238.

, Vida u obra de Petrarca. I. Lectura del Secretum, Padua und Chapel Hill-Barcelona 1974.

— , „Volontà e grazia nel Secretum“, in: Cardini, Roberto / Coppini, Donatella (Hgg.), Petrarca e Agostino, Roma 2004, 39-50.

Robson, Michael, „The Impact of the Cur Deus homo on the Early Franciscan School“, in: Luscombe / Evans, 334-347.

Rochais, Henri-Marie, Art. „Florilèges spirituels. I. Florilèges latins“, DictSpir 5 (1964), 435-460.

Röcke, Werner, Art. „Salomon und Markolf“, Enzyklopädie des Märchens 11 (2004), 1078-1085.

Roesch, Sophie, „Les emplois de verbum et sermo dans les expressions à verbe support verba facere, verba habere et sermonem habere", in: Moussy, Claude (Hg.), De lingva latina novae quaestiones. Actes du Xè Colloque International de Linguistique Latine. Paris-Sèvres, 19-23 avril 1999, Leuven 2001, 859-874.

Ronquist, Eyvind C., „Learning and Teaching in Twelfth-Century Dialogues“, Res publica litterarum 13 (1990), 239-256.

Rouse, Mary A. / Rouse, Richard H., „Florilegia of Patristic Texts“, in: Les genres littéraires, 165-180.

Ruelle, Pierre, Le Dialogue des Créatures. Traduction par Colart Mansion (1482) du Dialogus creaturarum (XIV siècle), Bruxelles 1985.

Ruhe, Ernstpeter (Hg.), Elucidarium und Lucidaires. Zur Rezeption des Werks von Honorius Augustodunensis in der Romania und in England, Wiesbaden 1993.

—, „Praedicatio et translatio. Das Elucidarium in der altfranzösischen Predigt“, in: Ruhe, Elucidarium, 9-28.

Rühl, Marco, Arguing and Communicative Asymmetry. The Analysis of the Interactive Process of Arguing in Non-Ideal Situations, Frankfurt a. M. u. a. 2002.

Santagata, Marco, I frammenti dell'anima. Storia e racconto nel „Canzoniere“ di Petrarca, Bologna 1992.

Santiago-Otero, Horacio (Hg.), Diálogo filosófico-religioso entre cristianismo, judaísmo e islamismo durante la Edad Media en la Península Ibérica, Turnhout 1994.

Santiago-Otero, Horacio / Reinhardt, Klaus, „Los Dialogi de Pedro Alfonso: tradición manuscrita e impresa“, Azafea 1 (1985), 33-43.

Schaller, Dieter, „Das mittelalterliche Epos im Gattungssystem“, in: Erzgräber, Kontinuität und Transformation, 355-371 (jetzt auch in: Schaller, Studien, 296-312 und Nachtrag 428-429).

— , „Gattungs- und Formtypen in den ,Carmina Burana amatoria“ “, Mittellateinisches Fahrbuch 36 (2001), 77-93.

- ,Vergil und die Wiederentdeckung des Epos im frühen Mittelalter“, Medioevo e Rinascimento 1 (1987), 75-100, ND in: Schaller, Studien, 270-295 und Nachtrag 428.

—, Studien zur lateinischen Dichtung des Frühmittelalters, Stuttgart 1995.

Schimmelpfennig, Bernhard, „Fragen an Maria. Überlegungen zu einem bemerkenswerten Text aus S. Maria in Trastevere“, in: Felten / Jaspert, 857-864.

Schindel, Ulrich, „Wechselwirkungen zwischen Autoren-Kommentar und systematischem Lehrbuch“, in: Geerlings, Wilhelm / Schulze, Christian (Hgg.), Der Kommentar in Antike und Mittelalter. Beiträge zu seiner Erforschung, Leiden u. a. 2002, 103-118.

Schmidt, Paul Gerhard, „Die Erscheinung der toten Geliebten“, Zeitschrift fïr deutsches Altertum und deutsche Literatur 105 (1976), 99-111.

_ , „I conflictus“, in: Cavallo, Guglielmo / Leonardi, Claudio / Menestò, Enrico (Hgg.), Lo spazio letterario del Medioevo. 1. Il Medioevo latino. Volume I. La produzione del testo. Tomo II, Roma 1993, 157-169.

— , „Jubel und Resignation. Amtsjubiläen und Amtsniederlegungen von Bischöfen 
und Äbten in literarischen Texten des Mittelalters", Historische Zeitschrift 252 (1991), $541-557$.

, „Schreiben als monastische Konfliktbewältigung“, in: Fludernik, Monika / Gehrke, Hans-Joachim (Hgg.), Grenzgänger zwischen den Kulturen, Würzburg 1999, 157-162.

— _ „Von der Allgegenwart der Dämonen. Die Lebensängste des Zisterziensers Richalm von Schöntal“, Literaturwissenschaftliches Fahrbuch der Görresgesellschaft 36 (1995), 339-346.

Schmidt, Peter Lebrecht, „Die Rezeption des römischen Freundschaftsbriefes (Cicero Plinius) im frühen Humanismus (Petrarca - Coluccio Salutati)“, in: Worstbrock, Franz Josef (Hg.), Der Brief im Zeitalter der Renaissance, DFG Mitteilung der Kommission für Humanismusforschung 9, Weinheim 1983, 25-50 (jetzt auch in: Schmidt, Peter Lebrecht, Traditio latinitatis. Studien zur Rezeption und Überlieferung der lateinischen Literatur, Stuttgart 2000, 142-165).

__ , „Zur Typologie und Literarisierung des frühchristlichen lateinischen Dialogs“, in: Fuhrmann, Manfred (Hg.), Christianisme et formes littéraires de l'Antiquité tardive en Occident, Génève 1976, 101-190.

Schmitt, Jean-Claude, La conversion d'Hermann le Fuif. Autobiographie, histoire et fiction, Paris 2003.

Schmitz, Wolfgang, „Die Kölner Ausgaben des Dialogus inter clericum et militem in buchgeschichtlicher Sicht“, Gutenberg-Fahrbuch 74 (1996), 106-112.

Schönbach, Anton, Über Gutolf von Heiligenkreuz. Untersuchungen und Texte, Sitzungsberichte der Kaiserlichen Akademie der Wissenschaften in Wien. Philosophisch-Historische Klasse 150, Wien, 1904.

Schreckenberg, Heinz, Die Juden in der Kunst Europas, ein historischer Bildatlas, Göttingen / Freiburg u. a. 1996.

Schrimpf, Gangolf, Art. „Johannes Scottus Eriugena“, TRE 17 (1988), 156-172.

Schürer, Markus, Das Exemplum oder die erzählte Institution. Studien zum Beispielgebrauch bei den Dominikanern und Franziskanern des 13. Fahrhunderts, Münster u. a. 2005.

Seelhorst, Jörg, Autoreferentialität und Transformation. Zur Funktion mystischen Sprechens bei Mechthild von Magdeburg, Meister Eckhart und Heinrich Seuse, Tübingen / Basel 2003.

Seidensticker, Bernd, Die Gesprächsverdichtung in den Tragödien Senecas, Heidelberg 1969.

Seiferth, Wolfgang, Synagoge und Kirche im Mittelalter, München 1964.

Seit, Stefan, „Abaelards Gespräch und die Grenzen einer rationalen Gotteslehre“, in: Lutz-Bachmann / Fidora, 40-95

Serper, Arié, „Le Débat entre Synagogue et Église au XIII ${ }^{\mathrm{e}}$ siècle“, Revue des études juives 4 ser. 3 (1964), 307-333.

Severus, Emmanuel von / Solignac, Aimé, Art. „Méditation. I. De l'Écriture aux auteurs médiévaux“, DictSpir 10 (1980), 906-914.

Shanzer, Danuta, „Laughter and humour in the early medieval Latin west“, in: Halsall, Humour, 25-47.

Sharpe, Richard, Titulus. Identifying Medieval Latin Texts. An Evidence-Based Approach, Turnhout 2003.

Silagi, Gabriel, Art. „Clemens Scotus“, LMA 2 (1983), 2149.

Siniscalco, Paolo, Art. „Tractatus“, Dizionario patristico e di antichità cristiane, Bd. 2 (1984), 3491.

Smet, Joachim, The Carmelites. A History of the Brothers of our Lady of Mount Carmel. Ca. 1200 until the Council of Trent, Roma 1975.

Soler i Llopart, Albert, „El Liber super psalmum quicumque de Ramon Llull i l'opció pels tàrtars“, Studia Lulliana 32 (1992), 3-19.

Solignac, Aimé, Art. „Werner de Ratisbonne“, DictSpir 16 (1994), 1369-1371.

Southern, Richard W., Saint Anselm: a portrait in a landscape, Cambridge 1990 (6. unver. Auflage 2004).

_ , „St. Anselm and his English pupils“, Medieval and Renaissance Studies 1 (1941/43, ND 1975), 3-34. 
Southern, Richard W. / Schmitt, Franz S., Memorials of St. Anselm, Auctores Britannici Medii Aevi 1, Oxford 1969.

Spanneut, Michel, Art. „Sénèque“, DictSpir 14 (1990), 570-598.

Speer, Andreas, Die entdeckte Natur: Untersuchungen zu Begründungsversuchen einer ,scientia naturalis" im 12. Fahrhundert, Leiden u. a. 2005.

_, ,Ratione duce. Die naturphilosophischen Dialoge des Adelard von Bath und des Wilhelm von Conches“, in: Jacobi, Gespräche lesen, 199-229.

Speyer, Augustin, Kommunikationsstrukturen in Senecas Dramen. Eine pragmatisch-linguistische Analyse mit statistischer Auswertung als Grundlage neuer Ansätze zur Interpretation, Göttingen 2003.

Spinelli, Franco (Hg.), Albertano da Brescia. Alle origini del Razionalismo economico, dell'Umanesimo civile, della Grande Europa, Brescia 1996.

Stacey, Robert C., ,Crusades, Martyrdoms and the Jews of Norman England, 10961190“, in: Haverkamp, Fuden und Christen, 233-251.

Stelzer, Winfried, Gelehrtes Recht in Österreich. Von den Anfängen bis zum frühen 14. Jh., Wien / Köln / Graz 1982.

Stierle, Karlheinz / Warning, Rainer (Hgg.), Das Gespräch, München 1984.

Stirnimann, Heinrich, „Mystik und Metaphorik. Zu Seuses Dialog“, Freiburger Zeitschrift fir Philosophie und Theologie 25 (1978), 233-303.

Störmer-Caysa, Uta, „Heldendialoge in Biterolf und Dietleib“, in: Henkel / Jones / Palmer, 21-39.

Stotz, Peter, „Conflictus. Il contrasto poetico nella letteratura latina medievale“, in: Pedroni / Stäuble, 165-187.

_ _, „Dichten als Schulfach - Aspekte mittelalterlicher Schuldichtung“, Mittellateinisches Fahrbuch 16 (1981), 1-16.

—- Handbuch zur lateinischen Sprache des Mittelalters, Bd. 1-5, München 1996-2004.

Strothmann, Friedrich Wilhelm, Die Gerichtsverhandlung als literarisches Motiv in der deutschen Literatur des ausgehenden Mittelalters, Jena 1930.

Sturges, Robert S., „A Middle English Version of the Pseudo-Augustinian Soliloquies“, Manuscripta 29 (1985), 73-79.

Suchier, Walther, L'Enfant Sage (Das Gespräch des Kaisers Hadrian mit dem klugen Kinde Epitus), Dresden 1910.

Suerbaum, Almut, „Dialogische Identitätskonzeptionen bei Mechthild von Magdeburg“, in: Henkel / Jones / Palmer, 239-255.

Sweeney, Eileen, „Anselm und der Dialog. Distanz und Versöhnung“, in: Jacobi, Gespräche lesen, 101-124.

Szittya, Penn R., The Antifraternal Tradition in Medieval Literature, Princeton 1986.

Szövérffy, Joseph, Marianische Motivik der Hymnen. Ein Beitrag zur Geschichte der marianischen Lyrik im Mittelalter, Leiden 1985.

Tagliaferro, Eleonora, „Gli Hermeneumata. Testi scolastici di età imperiale tra innovazione e conservazione", in: Celentano, Maria Silvana (Hg.), Ars / Techne. Il manuale tecnico nelle civiltà greca e romana. Atti del Convegno Internazionale Università, G. d'Annunzio " die Chieti-Pescara 29-30 ottobre 2001, Alessandria 2003, 51-77.

Teeuwen, Mariken, The Vocabulary of Intellectual Life in the Middle Ages, Etudes sur le vocabulaire intellectuel du Moyen Age 10, Turnhout 2003.

Telle, Joachim, Art. „Alchemie“, LMA 1 (1980), 329-342.

Teske, Roland J., ,Augustine of Hippo and the Quaestiones et Responsiones Literature“, in: Volgers / Zamagni, 127-144.

Testard, Maurice, Art. „Cicero“, Augustinus-Lexikon 1 (1986-1994), 913-930.

- Saint Augustin et Cicéron, 2 Bde., Paris 1958.

Thilo, Hans-Joachim, Art. „Gespräch“, TRE 13 (1984), 147-151.

Tinkler, John F., „Humanism and Dialogue“, Parergon NS 6 (1988), 197-214.

Toch, Michael, Die Fuden im mittelalterlichen Reich, Enzyklopädie deutscher Geschichte 44, München 1998. 
Tolan, John, Petrus Alfonsi and his Medieval Readers, Gainesville (Florida) 1993.

Tophinke, Doris, „Zum Problem der Gattungsgrenze - Möglichkeiten einer prototypentheoretischen Lösung“", in: Frank / Haye / Tophinke, 161-182.

Tovar Paz, Francisco-Javier, „Empleo léxico de tractatus, sermo y homilia en Agustín de Hipona y Jerónimo de Estridón“, Excerpta philologica 4-5 (1994-1995), 439-446.

Troncarelli, Fabio, Cogitatio mentis. L'eredità di Boezio nell'Alto Medioevo, Napoli 2005.

- Boethiana aetas. Modelli grafici e fortuna manoscritta della „Consolatio Philosophiae" tra IX e XII secolo, Alessandria 1987.

Tuilier, A., „La condamnation du Talmud par les maîtres universitaires parisiens. Ses causes et ses conséquences politiques et idéologiques", in: Dahan, Le brûlement, 7-20.

Tunberg, Terence O., „Conrad of Hirsau and His Approach to the Auctores“, Medievalia et Humanistica 15 (1987), 65-94.

Turner, Ralph V., „Descendit Ad Inferos: Medieval Views on Christ's Descent into Hell and the Salvation of the Ancient Just", Fournal of the History of Ideas 27 (1966), 173-194.

Utley, Francis Lee, „VII. Dialogues, Debates and Catechisms“, in: Hartung, Albert E. (Hg.), A Manual of the Writings in Middle English, Bd. 3, New Haven (Connecticut) 1972.

Utz Tremp, Kathrin, Quellen zur Geschichte der Waldenser von Freiburg im Üchtland (13991439), MGH Quellen zur Geistesgeschichte des Mittelalters 18, Hannover 2000.

Valle Cintra, Maria Adelaide (Hg.), Livro de soliloquio de sancto Agostinho (Cód. Alcob. CCLXXIII 198), Lisboa 1957.

Valois, Noël, La France et le Grand Schisme d'Occident, 4 Bde., Paris 1896-1902.

Van den Eynde, Damien, L'euvre littéraire de Géroch de Reichersberg, Roma 1957.

Van der Horst, Pieter W., „Philo and the Rabbis on Genesis: Similar Questions, Different Answers", in: Volgers / Zamagni, 55-70.

Van der Toorn, Karel, ,,The Ancient Near Eastern Literary Dialogue as a Vehicle of Critical Reflection“, in: Reinink / Vanstiphout, 59-75.

Van Fleteren, Frederick, „Augustine's Influence on Anselm's Proslogion“, in: Luscombe / Evans, 56-69.

Vandenbroucke, François, Pour l'histoire de la théologie morale. La morale monastique du XI au XVI siècle, Analecta medievalia namurcensia 20, Louvain / Lille 1966.

Vauchez, André, „Die Bettelorden und ihr Wirken in der städtischen Gesellschaft“, in: Vauchez, André (Hg.), Die Geschichte des Christentums. Religion, Politik, Kultur. Bd. 5. Machtfille des Papsttums (1054-1274), deutsche Ausgabe bearb. und hrsg. von Odilo Engels unter Mitarbeit von Georgios Makris und Ludwig Vones, Freiburg / Basel / Wien 1994, 833-860 (französische Originalausgabe: Histoire du christianisme des origines à nos jours. Tome V: Apogée de la papauté et expansion de la chrétienté (1054-1274), Paris 1993).

Vennebusch, Joachim, „Unbekannte Miracula des Caesarius von Heisterbach“, Annalen des historischen Vereins fiir den Niederrhein 184 (1981), 7-19.

Vernant, Denis, „Dialectique, forme dialogale et dialogique“, in: Luzzati, Daniel / Beacco, Jean-Claude / Mir-Samii, Reza / Murat, Michel / Vivet, Martial (Hgg.), Le Dialogique. Colloque international sur les formes philosophiques, linguistiques, littéraires, et cognitives du dialogue. Université du Maine, 15-16 septembre 1994, Bern u. a. 1997, 11-27.

Vicaire, Marie-Humbert, „Contra fudaeos meridionaux au début du XIII ${ }^{\mathrm{e}}$ siècle. Alain de Lille, Évrard de Béthune, Guillaume de Bourges“, in: Vicaire / Blumenkranz, Juifs et judaïsme, 269-293.

Vicaire, Marie-Humbert / Blumenkranz, Bernhard (Hgg.), Fuifs et judaïsme au Languedoc, Toulouse 1977.

Villa, Claudia, „I Classici“, in: Cavallo, Guglielmo / Leonardi, Claudio / Menestò, Enrico (Hgg.), Lo spazio letterario del Medioevo. 1. Il Medioevo latino. 1.1 La produzione del testo, Roma 1992, 479-522. 
Villa, Claudia, „Progetti letterari e ricezione europea di Albertano da Brescia“, in: Spinelli, Albertano da Brescia, 57-67.

Viola, Coloman, „Manières personnelles et impersonnelles d'aborder un problème: saint Augustin et le XII ${ }^{\mathrm{e}}$ siècle. Contribution à l'histoire de la quaestio", in: Les genres littéraires, 11-30.

Volgers, Annelie / Zamagni, Claudio (Hgg.), Erotapokriseis. Early Christian Question-andAnswer Literature in Context. Proceedings of the Utrecht Colloquium, 13-14 October 2003, Leuven u. a. 2004.

Volkmann, Laurenz, Art. „Dialogizität“, in: Nünning, Ansgar (Hg.), Metzler-Lexikon Literatur- und Kulturtheorie, Stuttgart-Weimar 1998, 92-93.

Vollmann, Benedikt Konrad, „Die Wiederentdeckung des Subjekts im Hochmittelalter“, in: Fetz, Reto Luzius / Hagenbüchle, Roland / Schulz, Peter (Hgg.), Geschichte und Vorgeschichte der modernen Subjektivität, Berlin / New York 1998, 380-393.

_, „Lateinisches Schauspiel des Mittelalters?“, in: Ziegeler, Hans-Joachim (Hg.), Ritual und Inszenierung. Geistliches und weltliches Drama des Mittelalters und der Frühen Neuzeit, Tübingen 2004, 1-8.

—, „Stil und Anspruch des Horologium sapientiae“, in: Blumrich / Kaiser, 84-93.

Voss, Bernd R., Der Dialog in der frühchristlichen Literatur, München 1970.

Waardenburg, Jacques / Limor, Ora / Dingel, Irene, Art. „Religionsgespräche“, TRE 28 (1997), 631-681.

Wachinger, Burghart, Art. „Secundus“, VL 11 (2004), 1402-1408.

Walsh, Katherine, A Fourteenth-Century Scholar and Primate: Richard FitzRalph in Oxford, Avignon and Armagh, Oxford 1981.

Walther, Hans, Das Streitgedicht in der lateinischen Literatur des Mittelalters, München 1920 (Neudruck mit Vorwort, Nachträgen und Register von Paul Gerhard Schmidt, Hildesheim 1984).

Weber, Dorothea, Art. „Dialog“, Lexikon der antiken christlichen Literatur, 3. neubearbeitete und erweiterte Auflage Freiburg i. Br. 2002, 191-193.

Weber, Gerd Wolfgang (Hg.), Idee - Gestalt - Geschichte. Festschrift Klaus von See, Odense 1988.

Weigand, Edda, „Discourse, Conversation, Dialogue“, in: Weigand, Edda (Hg.), Concepts of Dialogue. Considered from the Perspective of Different Disciplines, Tübingen 1994, $49-75$.

- , ,The Dialogic Action Game“, in: Coulthard, Malcolm / Cotterill, Janet / Rock, Frances (Hgg.), Dialogue Analysis VII: Working with Dialogue. Selected Papers from the 7th IADA Conference Birmingham 1999, Tübingen 2000, 1-18.

Weijers, Olga, La ,disputatio 'à la Faculté des arts de Paris (1200-1350 environ). Esquisse d'une typologie, Studia artistarum 2, Turnhout 1995.

Weimar, Peter, „Die legistische Literatur der Glossatorenzeit“, in: Coing, Helmut (Hg.), Handbuch der Quellen und Literatur der neueren europäischen Privatrechtsgeschichte. 1. Band. Mittelalter (1100-1500). Die Gelehrten Rechte und die Gesetzgebung, München 1973, 129-260.

Weisweiler, Heinrich, „Hugos von St. Viktor Dialogus de sacramentis legis naturalis et scriptae als frühscholastisches Quellenwerk“", in: Miscellanea Giovanni Mercati. Volume II. Letteratura medioevale, Città del Vaticano 1946, 179-219.

Welter, Jean Thiébaut, L'exemplum dans la littérature religieuse et didactique du moyen âge, Paris / Toulouse 1927 (ND Genève 1973).

Werner, Hans-Joachim, ,Meliores viae sophiae. Alkuins Bestimmungen der Philosophie in der Schrift Disputatio de vera philosophia“, in: Aertsen, Jan A. / Speer, Andreas (Hgg.), Was ist Philosophie im Mittelalter?, Berlin / New York 1998, 452-459.

Westermann, Hartmut, „Wahrheitssuche im Streitgespräch. Überlegungen zu Peter Abaelards Dialogus inter philosophum, judaeum et christianum." In: Jacobi, Gespräche lesen, 157-197.

Wiesenbach, Joachim, „Wilhelm von Hirsau. Astrolab und Astronomie im 11. Jahrhundert“ (Mit einem Nachtrag von Renate Neumüllers-Klauser: „Zu den Buchstaben- 
formen der Inschrift auf der Sphaera des Wilhelm von Hirsau in Regensburg"), in: Schreiner, Klaus (Hg.), Hirsau St. Peter und Paul 1091-1991. Teil II. Geschichte, Lebens- und Verfassungsformen eines Reformklosters, Stuttgart 1991, 109-156.

Wilmart, André, Auteurs spirituels et textes dévots du Moyen Âge latin: études d'histoire littéraire, Paris 1932 (ND Paris 1971).

Wilson, Kenneth J., Incomplete Fictions. The Formation of English Renaissance Dialogue, Washington 1985.

, , ,The Continuity of Post-Classical Dialogue“, Cithara 21 (1981), 23-44.

Witt, Ronald G., In the footsteps of the ancients: the origins of humanism from Lovato to Bruni, Leiden / Boston / Köln 2000.

Wolf, Monika, ,so tünd ich dir verbinden din ougen ond brich dir din baner ouch en zweÿ. Ecclesia und Synagoge in fortwährendem Streit", in: Schulze, Ursula (Hg.), fuden in der deutschen Literatur des Mittelalters. Religiöse Konzepte - Feindbilder - Rechtfertigungen, Tübingen 2002, 35-58.

Wood, Rega, „Richard Rufus of Cornwall and Aristotle's Physics“, Franciscan Studies 52 (1992), 247-281.

Worstbrock, Franz Josef, Art. „Isidor von Sevilla“, VL 11 (2004), 717-746.

, Art. „Warnerius von Basel“, VL 10 (1999), 726-733.

Art. „Wilhelm von Hirsau“, VL 10 (1999), 1100-1110.

Worstbrock, Franz Josef / Klaes, Monika / Lütten, Jutta, Repertorium der Artes dictandi des Mittelalters. Teil 1. Von den Anfängen bis um 1200, München 1992.

Wright, Thomas, The latin poems commonly attributed to Walter Mapes, London 1841.

Zamagni, Claudio, „Une introduction méthodologique à la littérature patristique des questions et réponses: le cas d'Eusèbe de Césarée“, in: Volgers / Zamagni, 7-24.

Ziolkowski, Jan, „The deeds and words of Aesop and Marcolf“, in: Walz, Dorothea (Hg.), Scripturus vitam. Lateinische Biographie von der Antike bis in die Gegenwart. Festgabe fir Walter Berschin zum 65. Geburtstag, Heidelberg 2002, 105-123.

Zöckler, Otto, Der Dialog im Dienste der Apologetik, Gütersloh 1894 (auch erschienen in Beweis des Glaubens 29 = NF 14 (1893), 209-230, 301-315, 331-349, 386-396). 